\title{
Incorporation of QCD effects in basic corrections of the electroweak theory
}

\author{
Sergio Fanchiotti
}

Theory Division, CERN, 1211 Geneva 23, Switzerland

\author{
Bernd Kniehl \\ II. Institut für Theoretische Physik, Universität Hamburg, Luruper Chaussee 149, 2000 Hamburg 50, Germany \\ Alberto Sirlin \\ Department of Physics, New York University, 4 Washington Place, New York, New York 10003
}

(Received 23 December 1993)

\begin{abstract}
We study the incorporation of QCD effects in the basic electroweak corrections $\Delta \hat{r}, \Delta \hat{r}_{W}$, and $\Delta r$. They include perturbative $O\left(\alpha \alpha_{s}\right)$ contributions and $t \bar{t}$ threshold effects. The latter are studied in the resonance and Green-function approaches, in the framework of dispersion relations that automatically satisfy relevant Ward identities. Refinements in the treatment of the electroweak corrections, in both the modified minimal subtraction $(\overline{\mathrm{MS}})$ and the on-shell schemes of renormalization, are introduced, including the decoupling of the top quark in certain amplitudes, its effect on $\hat{e}^{2}\left(m_{Z}\right)$ and $\sin ^{2} \hat{\theta}_{W}\left(m_{Z}\right)$, the incorporation of recent results on the leading irreducible $O\left(\alpha^{2}\right)$ corrections, and simple expressions for the residual, i.e., "nonelectromagnetic," parts of $\Delta \hat{r}, \Delta \hat{r}_{W}$, and $\Delta r$. The results are used to obtain accurate values for $m_{W}$ and $\sin ^{2} \hat{\theta}_{W}\left(m_{Z}\right)$, as functions of $m_{t}$ and $m_{H}$. The higher-order effects induce shifts in these parameters comparable to the expected experimental accuracy, and they increase the prediction for $m_{t}$ derived from current measurements. The $\overline{\mathrm{MS}}$ and the on-shell calculations of $\Delta r$, in a recently proposed formulation, are compared and found to be in excellent agreement over the wide ranges $60 \mathrm{GeV} \leq m_{H} \leq 1 \mathrm{TeV}, m_{Z} \leq m_{t} \leq 250 \mathrm{GeV}$.
\end{abstract}

PACS number(s): 11.10.Gh, 12.10.Dm, 12.38.-t, 14.80.Er

\section{INTRODUCTION}

It has been recently shown [1] that the modified minimal subtraction $(\overline{\mathrm{MS}})$ method of renormalization provides a very convenient framework to incorporate higher-order corrections to $\Delta r[2]$ arising from resummation of one-loop effects. These include not only leading logarithms of $O\left(\left[\alpha \ln \left(m_{Z} / m_{f}\right)\right]^{n}\right)$, where $m_{f}$ is a generic fermion mass, and subleading logarithms of $O\left(\alpha^{2} \ln \left(m_{Z} / m_{f}\right)\right)$ [3], but also terms of $O\left(\left(\alpha m_{t}^{2} / m_{W}^{2}\right)^{n}\right)$ $[1,4,5]$. The reason can be traced to the fact that in this method one essentially subtracts the divergent parts of the amplitudes. In contrast with other approaches, this procedure circumvents the introduction of mass singularities and $O\left(\alpha m_{t}^{2} / m_{W}^{2}\right)$ terms via the finite parts of the counterterms. As a consequence, the renormalized perturbative expansion has a structure very similar to that of the bare theory, where resummation of one-loop effects is easy to implement $[1,4]$. There are, of course, irreducible two-loop contributions of $O\left(\left(\alpha m_{t}^{2} / m_{W}^{2}\right)^{2}\right)$. As discussed in Refs. $[1,4,5]$, these can be gleaned from Ref. [6] and the more recent work of Ref. [7] on the $O\left(\alpha^{2}\right)$ corrections to the $\rho$ parameter.

It has also been recently shown [8] that it is possible to derive a simple and accurate expression for $\Delta r$, within the on-shell method of renormalization [2], which contains the same leading and subleading contributions described above. On the other hand, the irreducible twoloop contributions of $O\left(\alpha^{2} m_{t}^{2} / m_{W}^{2}\right)$ have not been com- puted, so that both the $\overline{\mathrm{MS}}$ and on-shell calculations of $\Delta r$ become uncertain at this level of accuracy. In fact, one can see that the difference between the two calculations and their inherent theoretical uncertainty due to the neglect of higher-order electroweak corrections start with subleading terms of $O\left(\left(\alpha / \pi s^{2}\right)\left(c^{2} / s^{2}\right) x_{t}\right)$, where $x_{t}$ is the leading correction to the $\rho$ parameter [cf. Eq. (17b)], or, equivalently, of $O\left(\left(c^{2} / s^{2}\right)\left(\alpha_{2} / 2 \pi\right)\left(\kappa_{t} / 2 \pi\right)\right)$, where $\alpha_{2} \equiv$ $g^{2} / 4 \pi$ and $\kappa_{t} \equiv\left(G_{t}\right)^{2} / 4 \pi$ are the SU(2) and the Yukawa couplings of the top quark, respectively. These are very small for low $m_{t}$ and are expected to be $\approx 8 \times 10^{-4}$ for $m_{t}=250 \mathrm{GeV}$. (As we will see, over the large ranges $m_{Z} \leq m_{t} \leq 250 \mathrm{GeV}, 60 \mathrm{GeV} \leq m_{H} \leq 1 \mathrm{TeV}$, the actual numerical evaluation of the on-shell expression of Ref. [8] and the $\overline{\mathrm{MS}}$ calculations show a very small difference, reaching a maximum value of $2.5 \times 10^{-4}$ at $m_{t}=250 \mathrm{GeV}$ and $m_{H}=1 \mathrm{TeV}$, a very precise agreement which may be somewhat fortuitous.) This uncertainty is to be compared with an estimated error of $\pm 9 \times 10^{-4}$ originating in the $O(\alpha)$ contributions of the first five quark flavors $[9,10]$.

In order to set the stage for our discussion, it is convenient to recall at this point some of the basic relations of the on-shell and $\overline{\mathrm{MS}}$ frameworks $[1-3,11,12]$,

$$
s^{2}=\frac{A^{2}}{m_{W}^{2}(1-\Delta r)}
$$




$$
\begin{aligned}
\hat{s}^{2} & =\frac{A^{2}}{m_{W}^{2}\left(1-\Delta \hat{r}_{W}\right)}, \\
\hat{s}^{2} \hat{c}^{2} & =\frac{A^{2}}{m_{Z}^{2}(1-\Delta \hat{r})},
\end{aligned}
$$

where $m_{W}$ and $m_{Z}$ are the physical masses, $A=$ $\left[\pi \alpha /\left(\sqrt{2} G_{\mu}\right)\right]^{1 / 2}=(37.2802 \pm 0.0003) \mathrm{GeV}, s^{2}, \hat{s}^{2}$, and $\hat{c}^{2}$ are abbreviations for $\sin ^{2} \theta_{W} \equiv 1-m_{W}^{2} / m_{Z}^{2}$ and the $\overline{\mathrm{MS}}$ parameters $\sin ^{2} \hat{\theta}_{W}\left(m_{Z}\right)$ and $\cos ^{2} \hat{\theta}_{W}\left(m_{Z}\right)$, respectively, and $\Delta r, \Delta \hat{r}_{W}$, and $\Delta \hat{r}$ are radiative corrections. The 't Hooft mass $\mu$ has been set equal to $m_{Z}$ in Eqs. (2) and (3).

It follows from the analysis of Ref. [13] that $m_{Z}$ and $m_{W}$ in Eqs. (1) and (2) can be identified, phenomenologically, with the masses measured in current experiments and, theoretically, with the definition $m_{1}^{2}=m_{2}^{2}+\Gamma_{2}^{2}$, where $\bar{s}=m_{2}^{2}-i m_{2} \Gamma_{2}$ is the relevant complex pole position [14]. The latter is given by $\bar{s}=m_{0}^{2}+A(\bar{s})$, where $m_{0}$ is the bare mass and $A(s)$ the conventional self-energy, which includes tadpoles, tadpole counterterms, and, in the $Z^{0}$ case, $\gamma Z$ mixing effects that start in $O\left(\alpha^{2}\right)$. On general grounds, it is expected that $m_{2}$ and $\Gamma_{2}$, and therefore $m_{1}$, are gauge invariant to all orders $[13,15]$. Over a large class of gauges, including those in which explicit calculations have been carried out, $m_{1}$ differs from the "field-theoretic" or "on-shell" definition, $m^{2}=m_{0}^{2}+\operatorname{Re} A\left(m^{2}\right)$, by gauge-dependent terms of $O\left(\alpha^{3}\right)$ [13]. Because contributions of this order are well beyond the accuracy that may be achieved in the foreseeable future, the replacement of $m$ by the more rigorous definition $m_{1}$ does not require a modification of the radiative corrections $\Delta r, \Delta \hat{r}_{W}$, and $\Delta \hat{r}$. Using the expression

$$
s^{2}=\frac{1}{2}\left\{1-\left[1-\frac{4 A^{2}}{m_{Z}^{2}(1-\Delta r)}\right]^{1 / 2}\right\}
$$

equivalent to Eq. (1), the analogous one with $s^{2} \rightarrow \hat{s}^{2}$ and $\Delta r \rightarrow \Delta \hat{r}$, equivalent to Eq. (3), and the accurately known value $m_{Z}=(91.187 \pm 0.007) \mathrm{GeV}[16]$, the corrections $\Delta r$ and $\Delta \hat{r}$ lead to precise evaluations of $s^{2}$ and $\hat{s}^{2}$, as functions of $m_{t}$ and $m_{H}$. These, in turn, can be compared with other determinations of $s^{2}$ and $\hat{s}^{2}$ to constrain the value of $m_{t}$ and, in the future, that of $m_{H}$. They are also important input parameters in the prediction of the $Z^{0}$ partial widths and on-resonance asymmetries, as some of these observables depend very sensitively on the weak mixing angle (see, for example, Ref. [17]). More generally, the basic corrections $\Delta r, \Delta \hat{r}_{W}$, and $\Delta \hat{r}$ are frequently employed to verify the standard model (SM) at the level of its quantum corrections and in searches for signals of new physics [18-21]. It was also explained in Ref. [1] how $\Delta \hat{r}$ and $\Delta \hat{r}_{W}$, relevant corrections in the $\overline{\mathrm{MS}}$ framework, can be employed to evaluate the on-shell quantity $\Delta r$.

The aim of the present paper is to incorporate the leading QCD corrections in the calculations of the radiative corrections $\Delta r, \Delta \hat{r}$, and $\Delta \hat{r}_{W}$. We also introduce some refinements in our previous treatment of the electroweak corrections [1].

The relevant QCD contributions occur in the vacuum- polarization functions associated with the $W^{ \pm}$and $Z^{0}$ bosons and have been extensively discussed in the literature [22-31]. In particular, the QCD corrections involving the $(t, b)$ isodoublet are known to be significant for large $m_{t}$. There are actually two types of effects that may be classified as perturbative $O\left(\alpha \alpha_{s}\right)$ and threshold contributions. In the literature, the latter are frequently referred to as "nonperturbative." The perturbative $O\left(\alpha \alpha_{s}\right)$ components have been studied with two different methods: ( $i$ ) direct evaluation of the two-loop Feynman diagrams in dimensional regularization, an approach that goes back to the pioneering work of Djouadi and Verzegnassi [22]; (ii) calculation of the imaginary parts and computation of the full amplitude by means of suitably defined dispersion relations (DR's) [23-26]. It has been shown $[30,31]$ that the two approaches are equivalent in the evaluation of the perturbative contributions to $\Delta r$ and $\Delta \rho$, a welcome fact. On the other hand, the $\overline{\mathrm{MS}}$ scheme is implemented in the framework of dimensional regularization and, for a full determination of the subtraction constants, one must appeal to method (i).

Threshold effects on the absorptive parts of the selfenergies have, in turn, been discussed in two different approaches: (a) in Ref. [24] the contributions of densely spaced, narrow quarkonium resonances were taken into account on the basis of a specific quark-antiquark potential; $(b)$ in Refs. $[27,28]$ one considers the imaginary part of the Green function for the nonrelativistic Schrödinger equation that characterizes the $t \bar{t}$ system near threshold. The latter formulation effectively resums the contributions of soft-gluon exchanges in the ladder approximation (see also Ref. [29]).

For sufficiently low $m_{t}$ there should be, near threshold, a rich spectrum of distinct nonrelativistic states bound by strong long-range forces and the approach $(a)$ is very natural. For increasing $m_{t}$, however, the weak decay of a single top quark inside the bound states becomes important and, for $m_{t} \gtrsim 130 \mathrm{GeV}$, the partial width of $t \rightarrow W^{+} b$ is so large that the revolution period of a $t \bar{t}$ bound state would exceed its lifetime. As a consequence, the individual resonances lose their distinctiveness and are smeared out to a coherent structure [27-29]. In that regime, the Green-function method is more appropriate. In summary, one expects approaches $(a)$ and $(b)$ to be preferable for lower $(\lesssim 130 \mathrm{GeV})$ and higher $(\gtrsim 130 \mathrm{GeV})$ values of $m_{t}$, respectively. Both formulations deal directly with the absorptive parts of the amplitudes. To obtain the real parts it is then necessary to employ DR's. This was done in detail in Ref. [31] using DR's for the vacuum-polarization functions directly constructed from relevant Ward identities [30]. In conjunction with very plausible assumptions concerning the asymptotic behavior of the threshold effects for large $q^{2}$, this procedure leads to specific results for the real parts.

In Sec. VII, we compare four different calculations of $m_{W}$ and $\sin ^{2} \hat{\theta}_{W}\left(m_{Z}\right)$, as functions of $m_{t}$ and $m_{H}$ : (i) only electroweak corrections; ( $i i)$ electroweak plus perturbative $O\left(\alpha \alpha_{s}\right)$ corrections; (iii) the above, plus threshold effects in the resonance approach; $(i v)$ same as (iii) with threshold effects in the Green-function framework. This 
allows us to demonstrate the magnitude of the QCD corrections and, at the same time, to separate the threshold effects from the more established perturbative $O\left(\alpha \alpha_{s}\right)$ contributions. Although the two approaches to treat the threshold effects are certainly not identical, we find the encouraging and fortunate result that their overall effects on $m_{W}$ and $\sin ^{2} \hat{\theta}_{W}\left(m_{Z}\right)$ are quite close over the entire range $m_{Z} \leq m_{t} \leq 250 \mathrm{GeV}$.

As mentioned before, aside from incorporating the QCD effects in the relevant self-energies, we introduce some refinements in our treatment of the $O(\alpha)$ electroweak corrections. Conceptually, the most interesting modification is a slight change in the definition of the fundamental couplings $\hat{e}^{2}\left(m_{Z}\right)$ and $\sin ^{2} \hat{\theta}_{W}\left(m_{Z}\right)$ of the $\overline{\mathrm{MS}}$ scheme, which is introduced in order to make them essentially independent of heavy particles such as the top quark or unknown massive excitations. In the case of $\sin ^{2} \hat{\theta}_{W}\left(m_{Z}\right)$, we follow a convention recently proposed at the one-loop level by Marciano and Rosner [32, 33], and explain how to extend it when $O\left(\alpha \alpha_{s}\right)$ corrections are included. We emphasize that these modifications in the definitions of the fundamental $\overline{\mathrm{MS}}$ parameters do not affect, to the order of the calculations, the relations between physical observables because they are compensated by corresponding changes in the appropriate radiative corrections. A second change is that we use an updated calculation by Jegerlehner [10] for the contribution of the first five quark flavors to the photon vacuum-polarization function. A third modification is that we incorporate the very recent results of Ref. [7] concerning the irreducible two-loop corrections of $O\left(\left(\alpha m_{t}^{2} / m_{W}^{2}\right)^{2}\right)$. In the rest of the calculations, as we did in Ref. [1], we treat the $u, d$, and $s$ quarks as massless but we now include terms of $O\left(\alpha m_{f}^{2} / m_{W}^{2}\right)$, where $f=c, b, \tau, \ldots$. Although they are very small - they contribute to $\Delta r$ only at the $\lesssim 1 \times 10^{-4}$ level-their incorporation may facilitate detailed comparisons with calculations by other authors.

The plan of the paper is the following: in Sec. II, we discuss the definitions of the basic $\overline{\mathrm{MS}}$ parameters $\hat{e}^{2}\left(m_{Z}\right)$ and $\sin ^{2} \hat{\theta}_{W}\left(m_{Z}\right)$ in the presence of the $O\left(\alpha \alpha_{s}\right)$ corrections, with emphasis on the decoupling of heavy particles. In Sec. III, we incorporate the perturbative $O\left(\alpha \alpha_{s}\right)$ contributions in $\Delta \hat{r}_{W}$ and $\Delta \hat{r}$. We emphasize the important fact that the magnitude of the $O\left(\alpha \alpha_{s}\right)$ effects depends sensitively on the precise definition of $m_{t}$. Our detailed calculations, as well as the other papers in the literature, employ the "on-shell" definition of $m_{t}$. In the discussion we give a brief argument to indicate why this choice is useful and appropriate. In Sec. IV, we present a simple method to separate the residual, i.e., "nonelectromagnetic," parts of $\Delta \hat{r}, \Delta \hat{r}_{W}$, and $\Delta r$. In Sec. V, we include the perturbative $O\left(\alpha \alpha_{s}\right)$ corrections in the calculation of $\Delta r$ in the on-shell scheme, using the formulation of Ref. [8]. In Sec. VI, we discuss the incorporation of threshold effects in $\Delta \hat{r}, \Delta \hat{r}_{W}$, and $\Delta r$. We include estimates, based on a simple "Bohr-atom" model, of the contribution of the $1 S$ toponium resonance to the imaginary part of the self-energies. We find that this simple model gives values roughly similar to the calculations carried out with more realistic quark-antiquark potentials. In Sec. VII, we use the theoretical results to carry out precise calculations of $m_{W}$ and $\sin ^{2} \hat{\theta}_{W}\left(m_{Z}\right)$, in the manner explained before. We find that the QCD and other higher-order corrections induce shifts in $m_{W}$ and $\sin ^{2} \hat{\theta}_{W}\left(m_{Z}\right)$ comparable with the expected experimental precision. Interestingly, all of them increase the value of $m_{t}$ derived from current measurements. We also compare the $\overline{\mathrm{MS}}$ and on-shell calculation of $\Delta r$, in the formulation of Ref. [8]. We find that, as was the case in the absence of perturbative $O\left(\alpha \alpha_{s}\right)$ corrections, the $\overline{\mathrm{MS}}$ and on-shell calculations of $\Delta r$ are very close over a large range of $m_{t}$ and $m_{H}$ values. The Appendixes discuss basic expressions for the perturbative $O\left(\alpha \alpha_{s}\right)$ corrections, the effect of top-quark decoupling in $\Delta \hat{r}_{W}$ and $\Delta \hat{r}$, and the very small contribution from finite fermion masses.

\section{THE PARAMETERS $\hat{e}^{2}\left(m_{Z}\right)$ AND $\sin ^{2} \hat{\theta}_{W}\left(m_{Z}\right)$}

In our previous treatment [1] we defined these parameters, at the one-loop level, by simply subtracting from the radiatively corrected cofactors the terms involving

$$
\delta=\frac{1}{n-4}+\frac{1}{2}[\gamma-\ln (4 \pi)]
$$

and setting the 't Hooft mass scale, $\mu$, equal to $m_{Z}$. Because at one loop $\delta$ always occurs in combination with $-\ln \mu$, this is equivalent to subtracting only the pole terms, $(n-4)^{-1}$, rescaling $\mu$ according to

$$
\mu=\frac{\mu^{\prime} e^{\gamma / 2}}{(4 \pi)^{1 / 2}}
$$

and then setting $\mu^{\prime}=m_{Z}$. The second formulation can be conveniently generalized to higher-order corrections and one can define the $\overline{\mathrm{MS}}$ renormalization procedure as the subtraction of pole terms of the form $(n-4)^{-m}$, where $m$ is an integer $\geq 1$, and the identification of the rescaled 't Hooft parameter $\mu^{\prime}$ with the relevant mass scale, in this case $m_{Z}$. As is well known, the factor $e^{\gamma / 2}(4 \pi)^{-1 / 2}$ is appended in Eq. (5b) to cancel relatively large numerical constants that are an artifact of dimensional regularization [34].

In Ref. [1] we applied this procedure uniformly, independently of whether the top quark is more or less massive than $m_{Z}$. On the other hand, it is desirable to treat heavy particles, as much as possible, as decoupled. For example, when $m_{t}>m_{Z}$ it is convenient to subtract from the amplitude terms involving $\ln \left(m_{t} / m_{Z}\right)$ and to absorb them in the coupling constants. In Ref. [1] we did not follow this route for two reasons: $(i) \sin ^{2} \hat{\theta}_{W}\left(m_{Z}\right)$ appears as a cofactor in several important radiatively corrected amplitudes and it is not possible to absorb completely the $\ln \left(m_{t} / m_{Z}\right)$ terms occurring in all of them; (ii) some important relations, such as Eq. (3), contain terms proportional to $m_{t}^{2}$, which certainly do not decouple. Recently, however, Marciano and Rosner [32, 33] proposed to implement the decoupling idea, at the one-loop level, by absorbing in $\sin ^{2} \hat{\theta}_{W}\left(m_{Z}\right)$ all $\ln \left(m / m_{Z}\right)$ terms with $m>m_{Z}$ occurring in a specific amplitude, namely the $\gamma Z$ self-energy evaluated at $q^{2}=m_{Z}^{2}, \operatorname{Re} A_{\gamma Z}\left(m_{Z}^{2}\right)$. Here 
$m$ is the mass of the top quark or any unknown heavy particle with $m>m_{Z}$. With this convention, a heavy top or a heavy unknown particle decouples in the limit $m / m_{Z} \gg 1$ from the cofactor $\hat{\kappa}\left(q^{2}\right)$ multiplying $\hat{s}^{2}$ in most neutral-current processes; as a consequence, this parameter can effectively be determined from the onresonance asymmetries without hindrance from unknown "heavy physics."

We now explain how we implement the decoupling idea in the presence of the $O\left(\alpha \alpha_{s}\right)$ corrections. It is convenient to start with $\hat{e}^{2}\left(m_{Z}\right)$, which we frequently abbreviate as $\hat{e}^{2}$. We recall the relation between the bare charge $e_{0}$ and the conventional renormalized charge $e$,

$$
\begin{aligned}
& e^{2}=e_{0}^{2}\left\{1+e_{0}^{2} \Pi_{\gamma \gamma}^{(f)}(0)\right. \\
& \left.\quad+\frac{7 e_{0}^{2}}{8 \pi^{2}}\left[\frac{1}{n-4}+\ln \frac{m_{W}}{\mu^{\prime}}-\frac{1}{21}\right]\right\}^{-1}
\end{aligned}
$$

where $\Pi_{\gamma \gamma}^{(f)}(0)$ is the usual fermionic vacuum-polarization function evaluated at $q^{2}=0$ and the last term represents $O\left(e_{0}^{2}\right)$ bosonic contributions to charge renormaliza- tion that must be included in the SM. The latter can be gleaned, for example, from Ref. [2]. As explained in Refs. [1,2], because of the existence of mass singularities associated with the light quarks, it is not possible to calculate perturbatively the contribution of the first five quark flavors to $\Pi_{\gamma \gamma}^{(f)}(0)$. Calling this contribution $\Pi_{\gamma \gamma}^{(5)}(0)$, the problem is circumvented by writing $\Pi_{\gamma \gamma}^{(5)}(0)=\operatorname{Re}\left[\Pi_{\gamma \gamma}^{(5)}(0)-\Pi_{\gamma \gamma}^{(5)}\left(m_{Z}^{2}\right)\right]+\operatorname{Re} \Pi_{\gamma \gamma}^{(5)}\left(m_{Z}^{2}\right)$. The first term represents the five-flavor contribution to the renormalized photon vacuum-polarization function at $q^{2}=m_{Z}^{2}$ and can be evaluated using dispersion relations, experimental data on $e^{+} e^{-} \rightarrow$ hadrons and QCD corrections $[2,9,10]$. The presence of a large invariant momentum, $q^{2}=m_{Z}^{2}$, in the third term prevents the occurrence of fermionic mass singularities and, as a consequence, $\operatorname{Re} \Pi_{\gamma \gamma}^{(5)}\left(m_{Z}^{2}\right)$ can be analyzed perturbatively. The same is, of course, true for the leptonic and bosonic contributions and, because of its large mass, for the top contribution $\Pi_{\gamma \gamma}^{(t)}(0)$. Including irreducible two-loop contributions of $O\left(\hat{\alpha} \hat{\alpha}_{s}, \hat{\alpha}^{2}\right)$ to $\hat{e}^{2} \Pi_{\gamma \gamma}^{(f)}(0)$ arising from virtual gluon and photon interchanges, we obtain (see Appendix A)

$$
\begin{aligned}
\hat{e}^{2} \Pi_{\gamma \gamma}^{(f)}(0)= & \frac{2 \hat{\alpha}}{3 \pi} \sum_{l}\left[\ln \frac{\mu^{\prime}}{m_{l}}\left(1+\frac{3 \hat{\alpha}}{4 \pi}\right)+\frac{45 \hat{\alpha}}{32 \pi}\right]+\frac{8 \hat{\alpha}}{9 \pi}\left[\ln \frac{\mu^{\prime}}{m_{t}}\left(1+\frac{\hat{\alpha}_{s}}{\pi}+\frac{\hat{\alpha}}{3 \pi}\right)+\frac{15}{8}\left(\frac{\hat{\alpha}_{s}}{\pi}+\frac{\hat{\alpha}}{3 \pi}\right)\right] \\
& +\frac{\hat{\alpha}}{\pi} \sum_{q \neq t} Q_{q}^{2}\left[2 \ln \frac{\mu^{\prime}}{m_{Z}}\left(1+\frac{\hat{\alpha}_{s}}{\pi}+\frac{3 \hat{\alpha}}{4 \pi} Q_{q}^{2}\right)+f_{1}\left(r_{q}\right)+\left(\frac{\hat{\alpha}_{s}}{\pi}+\frac{3 \hat{\alpha}}{4 \pi} Q_{q}^{2}\right) f_{2}\left(r_{q}\right)\right] \\
& +\hat{e}^{2} \operatorname{Re}\left[\Pi_{\gamma \gamma}^{(5)}(0)-\Pi_{\gamma \gamma}^{(5)}\left(m_{Z}^{2}\right)\right]-\frac{\hat{\alpha}}{\pi} \frac{I}{(n-4)},
\end{aligned}
$$

where the $l$ and $q$ sums run over leptons and quarks, respectively, the color factor 3 is henceforth explicitly included, $r_{q}=m_{Z}^{2} /\left(4 m_{q}^{2}\right)$

$$
\begin{aligned}
f_{1}(r) & =\ln (4 r)-\left(2+\frac{1}{r}\right)\left(1-\frac{1}{r}\right)^{1 / 2} \cosh ^{-1} \sqrt{r}+\frac{5}{3}+\frac{1}{r} \\
& =\frac{5}{3}+\frac{3}{2 r}+O\left(\frac{\ln r}{r^{2}}\right), \\
f_{2}(r) & =\ln (4 r)+\frac{\operatorname{ReV}(r)}{r}-4 \zeta(3)+\frac{55}{12} \\
& =-4 \zeta(3)+\frac{55}{12}-\frac{3}{r} \ln (4 r)+O\left(\frac{\ln ^{2} r}{r^{2}}\right),
\end{aligned}
$$

$\zeta(3)=1.20206 \ldots, V_{1}(r)$ is a complicated function defined in Ref. [25], and $I=16 / 3+5 \hat{\alpha}_{s} /(3 \pi)+11 \hat{\alpha} /(9 \pi)$. The first and second terms in Eq. (7a) are the finite parts of the leptonic and top contributions, while the sum over $q$ is the finite part of the perturbative evaluation of $\hat{e}^{2} \operatorname{Re} \Pi_{\gamma \gamma}^{(5)}\left(m_{Z}^{2}\right)$. In the latter the terms proportional to $r_{q}^{-1}$ are extremely small and we can replace the functions $f_{1}(r)$ and $f_{2}(r)$ by their asymptotic values $f_{1}(\infty)=5 / 3$ and $f_{2}(\infty)=55 / 12-4 \zeta(3) \approx-0.22491$. The last term in Eq. (7a) represents the divergent part up to terms of $O\left(\hat{\alpha}^{2}, \hat{\alpha} \hat{\alpha}_{s}\right)$. As is well known, up to two loops $\Pi_{\gamma \gamma}\left(q^{2}\right)$ is linear in $(n-4)^{-1}$ and $\ln \mu^{\prime}$. We note that the cofactors of $(n-4)^{-1}$ are equal to those of $\ln \left(1 / \mu^{\prime}\right)$ at one loop and to one-half those of $\ln \left(1 / \mu^{\prime}\right)$ at two loops.

In order to obtain the relation between $\hat{e}^{2}$ and $e^{2}$, one writes $e_{0}^{2}=\hat{e}^{2} / \hat{Z}_{e}$ in Eq. (6), uses the counterterms present in $\hat{Z}_{e}$ to cancel the $(n-4)^{-1}$ terms in Eqs. (6) and (7a), and sets $\mu^{\prime}=m_{Z}$ in those equations. The mass scale employed in $\hat{\alpha}_{s}$ is discussed in greater detail at the end of this section. With the exception of the top quark, all the particles contributing to Eqs. (6) and (7a) are less massive than $m_{Z}$ and their contribution is retained. To implement decoupling in Eq. (6), we also subtract the finite top contribution when $m_{t}>m_{Z}$, so that $\hat{Z}_{e}$ in that case contains an additional finite counterterm and reads 


$$
\hat{Z}_{e}=1+\frac{\hat{\alpha}}{\pi}\left(I-\frac{7}{2}\right) \frac{1}{n-4}-\left.\hat{e}^{2} \Pi_{\gamma \gamma}^{(t)}(0)\right|_{\overline{\mathrm{MS}}} \theta\left(m_{t}-m_{Z}\right),
$$

where $\overline{\mathrm{MS}}$ denotes the "finite part" after the $\overline{\mathrm{MS}}$ renormalization has been carried out, i.e., the remainder after the $(n-4)$ poles have been subtracted and $\mu^{\prime}$ has been set equal to $m_{Z}$, and the superscript $(t)$ refers to the top-quark contribution. Specifically,

$$
\left.\hat{e}^{2} \Pi_{\gamma \gamma}^{(t)}(0)\right|_{\overline{\mathrm{MS}}}=\frac{8 \hat{\alpha}}{9 \pi}\left[\ln \frac{m_{Z}}{m_{t}}\left(1+\frac{\hat{\alpha}_{s}}{\pi}+\frac{\hat{\alpha}}{3 \pi}\right)+\frac{15}{8}\left(\frac{\hat{\alpha}_{s}}{\pi}+\frac{\hat{\alpha}}{3 \pi}\right)\right] .
$$

The term proportional to $(n-4)^{-1}$ in Eq. (7d) cancels the divergent parts in Eqs. (6) and (7a). The other term subtracts the finite top contribution, i.e., the second term in Eq. (7a), when $m_{t}>m_{Z}$. Because this contribution does not exactly vanish at $m_{t}=m_{Z}$, the above prescription leads to a small discontinuity at $m_{t}=m_{Z}$. An alternative that would ensure continuity would be to do the matching at the point where the finite top correction vanishes, which is $m_{t}=1.073 m_{Z}=97.8 \mathrm{GeV}$. However, because the decoupling at $m_{t}>m_{Z}$ is easy to implement and is analogous to what is done in some QCD calculations [35], we will adopt it as our convention.

As the mass range $m_{t}<91 \mathrm{GeV}$ has been excluded at the $95 \%$ confidence level [36], we will henceforth assume that $m_{t}>m_{Z}$, in which case Eqs. (6), (7a), and (7d) lead to

$$
e^{2}=\frac{\hat{e}^{2}}{1+(\hat{\alpha} / \pi) \Delta_{\gamma}}
$$

where

$$
\begin{aligned}
\Delta_{\gamma}= & \frac{7}{4} \ln c^{2}-\frac{1}{6}+\frac{2}{3} \sum_{l}\left[\ln \frac{m_{Z}}{m_{l}}\left(1+\frac{3 \hat{\alpha}\left(m_{Z}\right)}{4 \pi}\right)+\frac{45 \hat{\alpha}\left(m_{Z}\right)}{32 \pi}\right]+\frac{55}{27}+\left(\frac{11 \hat{\alpha}_{s}\left(m_{Z}\right)}{9 \pi}+\frac{35 \hat{\alpha}\left(m_{Z}\right)}{108 \pi}\right)\left(\frac{55}{12}-4 \zeta(3)\right) \\
& +4 \pi^{2} \operatorname{Re}\left[\Pi_{\gamma \gamma}^{(5)}(0)-\Pi_{\gamma \gamma}^{(5)}\left(m_{Z}^{2}\right)\right],
\end{aligned}
$$

and $c^{2}$ is an abbreviation for $\cos ^{2} \theta_{W} \equiv m_{W}^{2} / m_{Z}^{2}$. Solving for $\hat{e}^{2}$, we have

$$
\hat{e}^{2}=\frac{e^{2}}{1-(\alpha / \pi) \Delta_{\gamma}}
$$

In $(\alpha / \pi) \Delta_{\gamma}$ we have retained very small terms of $O\left(\alpha^{2}\right)$ arising from virtual-photon interchange. We have done this because of the analogy with gluon contributions and the fact that they contain interesting leptonic mass singularities. It should be understood, however, that this does not represent a complete $O\left(\alpha^{2}\right)$ calculation as there are other irreducible two-loop contributions of this order arising from the bosonic sector and from $W^{ \pm}, Z^{0}$, and $H$ interchanges in the fermionic sector.

Equation (8c) allows us to compute $\hat{e}^{2}$ in terms of $\alpha$, independently of $m_{t}$ or unknown particles heavier than $m_{Z}$. Using $m_{Z}=91.187 \mathrm{GeV}[16], m_{W}=80.22 \mathrm{GeV}$ $[37,38], \hat{\alpha}_{s}=\hat{\alpha}_{s}\left(m_{Z}\right)=0.118$ [39], and $e^{2} \operatorname{Re}\left[\Pi_{\gamma \gamma}^{(5)}(0)-\right.$ $\left.\Pi_{\gamma \gamma}^{(5)}\left(m_{Z}^{2}\right)\right]=0.0282 \pm 0.0009[10]$, we find $(\alpha / \pi) \Delta_{\gamma}=$ $0.0668 \pm 0.0009$ or $\hat{\alpha}^{-1}=\left(4 \pi / \hat{e}^{2}\right)=127.9 \pm 0.1$. The expression for $(\alpha / \pi) \Delta_{\gamma}$ differs from the quantity $-2 \delta e /\left.e\right|_{\overline{\mathrm{MS}}}$ in Ref. [1] by the exclusion of the $\ln \left(m_{t} / m_{Z}\right)$ terms, a more accurate description of the QCD corrections, and the updated calculation of the five-flavor contribution. Numerically, however, $\hat{\alpha}^{-1}$ is very close to the value $127.8 \pm 0.1$ reported in Ref. [1] for $m_{t}=m_{Z}$, the small difference essentially arising from the change in the fiveflavor contribution [10].

Concerning $\hat{s}^{2} \equiv \sin ^{2} \hat{\theta}_{W}\left(m_{Z}\right)$, we recall that in the neutral-current amplitudes this parameter is multiplied by the electroweak form factor $\hat{\kappa}$, which contains the $\gamma Z$ mixing term $-(\hat{c} / \hat{s}) A_{\gamma Z}\left(q^{2}\right) / q^{2}[17,40]$. Here $A_{\gamma Z}\left(q^{2}\right)$ is the unrenormalized $\gamma Z$ transverse mixing amplitude as defined in Ref. [41], expressed in terms of the $\overline{\mathrm{MS}}$ couplings $\hat{e}, \hat{s}$, and $\hat{c}$. In order to implement the decoupling, we apply the Marciano-Rosner convention $[32,33]$, according to which the $\ln \left(m_{t} / m_{Z}\right)$ terms in $\operatorname{Re} A_{\gamma Z}\left(m_{Z}^{2}\right) / m_{Z}^{2}$ are subtracted for $m_{t}>m_{Z}$. At the two-loop level there is also an $m_{t}$-independent term, which must be subtracted, too. More generally, the idea is to subtract all contributions to $\operatorname{Re} A_{\gamma Z}\left(m_{Z}^{2}\right) / m_{Z}^{2}$ that involve particles of mass $m>m_{Z}$ and do not decouple in the limit $m \rightarrow \infty$. This can be implemented by adding a finite counterterm in the $\hat{s}^{2}$ renormalization, in analogy with Eq. (7d). Up to terms of $O\left(\alpha \alpha_{s}\right)$ we find, in the case of the top quark,

$$
s_{0}^{2}=\hat{s}^{2}\left\{1+\frac{\text { const }}{n-4}+\left.\frac{\hat{c}}{\hat{s}}\left(A_{\gamma Z}^{(t)}\right)^{\prime}(0)\right|_{\overline{\mathrm{MS}}}\right\},
$$

where $s_{0}^{2}$ is the bare parameter, $\left(A_{\gamma Z}^{(t)}\right)^{\prime}(0)=$ $\left.\left(d / d q^{2}\right) A_{\gamma Z}^{(t)}\left(q^{2}\right)\right|_{q^{2}=0}$, and $\overline{\mathrm{MS}}$ and the superscript $(t)$ have the same meaning as in Eq. (7d). Again, const/ $n-$ 4 ) is the divergent part of the counterterm and the last term is a finite contribution necessary to implement the decoupling of the $\ln \left(m_{t} / m_{Z}\right)$ and constant terms in the top contribution to $\operatorname{Re} A_{\gamma Z}\left(m_{Z}^{2}\right) / m_{Z}^{2}$. Specifically, we find: 


$$
\left.\frac{\hat{c}}{\hat{s}}\left(A_{\gamma Z}^{(t)}\right)^{\prime}(0)\right|_{\overline{M S}}=\frac{\hat{\alpha}}{\pi} d
$$

where

$$
d=\frac{1}{3}\left(\frac{1}{\hat{s}^{2}}-\frac{8}{3}\right)\left[\ln \frac{m_{t}}{m_{Z}}\left(1+\frac{\hat{\alpha}_{s}}{\pi}\right)-\frac{15 \hat{\alpha}_{s}}{8 \pi}\right] .
$$

In Eqs. (9a)-(9c) we have neglected all terms of $O\left(\alpha^{2}\right)$.

We must still discuss the mass scale employed in $\hat{\alpha}_{s}$. Following Refs. [22, 24-26, 31], in the present paper corrections arising from the $(t, b)$ isodoublet are computed with $\hat{\alpha}_{s}\left(m_{t}\right)$. The reason is that the dominant contributions due to this isodoublet involve mass scales of $O\left(m_{t}\right)$ $[31,42]$. This choice can also be justified by arguments based on effective-field theory [43]. On the other hand, the perturbative contributions from the two light-quark isodoublets involve self-energies evaluated at $q^{2}=m_{W}^{2}$ or $q^{2}=m_{Z}^{2}$ and, for that reason, are calculated with $\hat{\alpha}\left(m_{Z}\right)$. As an example, in Eqs. (7e) and (9c), which involve topquark contributions, we identify $\hat{\alpha}_{s}$ with $\hat{\alpha}_{s}\left(m_{t}\right)$, while in the perturbative part of Eq. (8b), which includes light- quark isodoublets, we employ $\hat{\alpha}_{s}\left(m_{Z}\right)$. [In the latter equation we make a slight and negligible change to the above isodoublet rule by also evaluating the very small bottom contribution with $\hat{\alpha}_{s}\left(m_{Z}\right)$.] Numerically, the finite counterterms in Eqs. (7d) and (9a) are quite small: for $m_{t}=150 \mathrm{GeV}$, they are $1.0 \times 10^{-3}$ in Eqs. (7d) and (7e) and $6.1 \times 10^{-4}$ in Eqs. (9b) and (9c); for $m_{t}=250 \mathrm{GeV}$, the corresponding values are $2.2 \times 10^{-3}$ and $1.5 \times 10^{-3}$, respectively. As we will see, when $\hat{s}^{2}$ is defined according to the decoupling convention explained above, the finite counterterm in Eq. (9a) introduces small compensatory shifts in $\Delta \hat{r}_{W}$ and $\Delta \hat{r}$. Similarly, $\hat{Z}_{e}$, defined in Eq. (7d), will introduce small compensatory changes in radiative corrections whenever $\hat{e}^{2}$ is employed as the zeroth-order parameter.

\section{PERTURBATIVE $O\left(\alpha \alpha_{s}\right)$ CORRECTIONS TO $\Delta \hat{r}_{W}, \Delta \hat{r}$, AND $\Delta r$}

When the decoupling of the top quark is implemented according to the discussion of Sec. II, the expression for $\Delta \hat{r}_{W}$ [cf. Eq. (2)] becomes (see Appendix B)

$$
\begin{aligned}
\Delta \hat{r}_{W}= & {\left[\frac{e^{2}}{\hat{s}^{2}} \operatorname{Re}\left(\frac{A_{W W}\left(m_{W}^{2}\right)-A_{W W}(0)}{m_{W}^{2}}\right)+e^{2} \Pi_{\gamma \gamma}^{(f)}(0)\right]_{\overline{\mathrm{MS}}}+\frac{\alpha}{\pi}\left(\frac{7}{4} \ln c^{2}-\frac{1}{6}\right) } \\
& +\frac{e^{2}}{16 \pi^{2} \hat{s}^{2}}\left\{6+\frac{\ln c^{2}}{s^{2}}\left[\frac{7}{2}-\frac{5}{2} s^{2}-\hat{s}^{2}\left(5-\frac{3 c^{2}}{2 \hat{c}^{2}}\right)\right]\right\}-\frac{\alpha}{\pi} d,
\end{aligned}
$$

where $A_{W W}\left(q^{2}\right)$ is the unrenormalized $W W$ transverse self-energy with the coupling $e^{2} / \hat{s}^{2}$ factored out [1], $d$ is defined in Eq. (9c), and the $\overline{\mathrm{MS}}$ symbol has the same meaning as in Sec. II. The quantities $\Delta \hat{r}_{W}, \Delta \hat{r}$, and $\Delta r$ are gauge invariant but some of their partial bosonic contributions are not. In Eq. (10a) and henceforth all explicit expressions and partial contributions are given in the 't Hooft-Feynman gauge. In particular, the expression involving curly brackets represents vertex- and box-diagram corrections to $\mu$ decay, evaluated in that gauge.

Except for the last term, Eq. (10a) is the same as Eq. (8b) in Ref. [1]. As $\hat{s}^{2}$ enters Eq. (2) as a zeroth-order parameter and is defined in the present paper according to the decoupling convention explained in Sec. II, there is now an additional contribution $-(\alpha / \pi) d$ arising from the finite contribution in Eqs. (9a)-(9c).

We now discuss the evaluation of Eq. (10a). The quantity $\left[e^{2} \Pi_{\gamma \gamma}^{(f)}(0)\right]_{\overline{M S}}$ can be obtained from Eq. (7a). We have

$$
\left[e^{2} \Pi_{\gamma \gamma}^{(f)}(0)\right]_{\overline{\mathrm{MS}}}+\frac{\alpha}{\pi}\left(\frac{7}{4} \ln c^{2}-\frac{1}{6}\right)=\frac{\alpha}{\pi}\left\{\Delta_{\gamma}+\frac{8}{9}\left[\ln \frac{m_{Z}}{m_{t}}\left(1+\frac{\hat{\alpha}_{s}}{\pi}+\frac{\hat{\alpha}}{3 \pi}\right)+\frac{15}{8}\left(\frac{\hat{\alpha}_{s}}{\pi}+\frac{\hat{\alpha}}{3 \pi}\right)\right]\right\}
$$

where $\Delta_{\gamma}$ is defined in Eq. (8b) and the other terms on the right-hand side (rhs) represent the top contribution. Inserting Eq. (10b) into Eq. (10a) and neglecting small terms of $O\left(\alpha^{2}\right)$, we obtain

$$
\Delta \hat{r}_{W}=\left[\frac{e^{2}}{\hat{s}^{2}} \operatorname{Re}\left(\frac{A_{W W}\left(m_{W}^{2}\right)-A_{W W}(0)}{m_{W}^{2}}\right)\right]_{\overline{\mathrm{MS}}}+\frac{\alpha}{\pi} \Delta_{\gamma}+\frac{e^{2}}{16 \pi^{2} \hat{s}^{2}}\left\{6+\frac{\ln c^{2}}{s^{2}}\left[\frac{7}{2}-\frac{5}{2} s^{2}-\hat{s}^{2}\left(5-\frac{3 c^{2}}{2 \hat{c}^{2}}\right)\right]\right\}-\frac{\alpha}{\pi} \hat{d}
$$

where

$$
\hat{d}=\frac{d}{1-8 \hat{s}^{2} / 3} .
$$

The bosonic contribution $\left(e^{2} / \hat{s}^{2} m_{W}^{2}\right)\left[A_{W W}^{(b)}\left(m_{W}^{2}\right)-A_{W W}^{(b)}(0)\right]_{\overline{\mathrm{MS}}}$ is given in Eq. (A.6) of Ref. [1]. In order to study the fermionic contributions, denoted by a superscript $(f)$, we define

$$
B^{(f)} \equiv \frac{e^{2}}{\hat{s}^{2}} \operatorname{Re}\left[\frac{A_{W W}^{(f)}\left(m_{W}^{2}\right)-A_{W W}^{(f)}(0)}{m_{W}^{2}}\right]_{\overline{\mathrm{MS}}}
$$


and write

$$
B^{(f)}=B_{0}^{(f)}+B_{\mathrm{QCD}}^{(f)},
$$

where $B_{0}^{(f)}$ and $B_{\mathrm{QCD}}^{(f)}$ stand for the $O(\alpha)$ and $O\left(\alpha \alpha_{s}\right)$ corrections. If we neglect very small terms proportional to $\alpha m_{q}^{2} / m_{W}^{2}(q=d, s, b)$, the mixing angles in the quark sector are irrelevant [41] and to zeroth order in $\alpha_{s}$ we have (cf. Eq. (A.5) of Ref. [1])

$$
B_{0}^{(f)}=\frac{\alpha}{2 \pi \hat{s}^{2}}\left\{2\left(\ln c^{2}-\frac{5}{3}\right)+\frac{\ln \omega_{t}}{2}+\frac{\omega_{t}}{8}\left(1+2 \omega_{t}\right)+\frac{\left(\omega_{t}-1\right)^{2}}{2}\left(1+\frac{\omega_{t}}{2}\right) \ln \left(1-\frac{1}{\omega_{t}}\right)\right\},
$$

where we have included the lepton and quark contributions and $\omega_{t}=m_{t}^{2} / m_{W}^{2}$. As a refinement, in Appendix C we discuss the contributions of $O\left(\mathrm{~m}^{2} / \mathrm{m}_{W}^{2}\right)$, where $m$ stands for a lepton or quark mass other than $m_{t}$. As these terms are very small, we may neglect in their evaluation the squares of the mixing angles. In that case one obtains a sum of isodoublet contributions, which, for arbitrary masses, is given in Appendix C. One finds that the corrections of $O\left(\mathrm{~m}^{2} / \mathrm{m}_{W}^{2}\right)$ to $B_{0}^{(f)}$ are indeed very small, of order $10^{-5}$.

The contribution of $O\left(\alpha \alpha_{s}\right)$ in Eq. (11b) is given by (see Appendix A)

$B_{\mathrm{QCD}}^{(f)}=\frac{\alpha}{4 \pi \hat{s}^{2}}\left\{\frac{2 \hat{\alpha}_{s}\left(m_{Z}\right)}{\pi}\left(\ln c^{2}+4 \zeta(3)-\frac{55}{12}\right)+\frac{\hat{\alpha}\left(m_{t}\right)}{\pi}\left[\ln c^{2}+4 \zeta(3)-\frac{55}{12}-4 \omega_{t}\left(F_{1}\left(1 / \omega_{t}\right)-F_{1}(0)\right)+\ln \omega_{t}\right]\right\}$

where $F_{1}(x)$ is defined in Ref. [25]. In Eq. (11d) we have neglected all quark masses other than $m_{t}$. The first term in Eq. (11d) arises from the $(u, d)$ and $(c, s)$ isodoublets, while the second is associated with the $(t, b)$ doublet. In Eqs. (10c), (10d), and (11d) the mass scale of $\hat{\alpha}_{s}$ has been chosen according to the prescription explained at the end of Sec. II.

The asymptotic behavior of $\Delta \hat{r}_{W}$ for large $m_{t}$ can be obtained from Eqs. (9c), (10c), (10d), (11c), and (11d) and is given by

$\Delta \hat{r}_{W} \sim \frac{\alpha}{6 \pi \hat{s}^{2}}\left(1+\frac{\hat{\alpha}_{s}\left(m_{t}\right)}{\pi}\right) \ln \frac{m_{t}}{m_{Z}} \quad\left(m_{t} \gg m_{Z}\right)$.

This exhibits a smaller coefficient than the corresponding expression in Ref. [12], a feature that is due to a partial cancellation with the finite counterterm $-(\alpha / \pi) d$ in Eq. (10a). The asymptotic behavior for $m_{H} \gg m_{Z}$ is the same as in Ref. [12], namely

$$
\Delta \hat{r}_{W} \sim \frac{\alpha}{24 \pi \hat{s}^{2}} \ln \frac{m_{H}}{m_{Z}} \quad\left(m_{H} \gg m_{Z}\right) .
$$

We now turn our attention to $\Delta \hat{r}$. When the top decoupling is implemented according to Sec. II, the expression for $\Delta \hat{r}$ becomes (see Appendix B)

$$
\begin{aligned}
\Delta \hat{r}=\Delta \hat{r}_{W} & -\frac{\hat{e}^{2}\left[1-\Delta \hat{r}_{W}-(\alpha / \pi) \hat{d}\right]}{\hat{s}^{2} m_{Z}^{2}} \\
\times & \operatorname{Re}\left[\frac{A_{W W}\left(m_{W}^{2}\right)}{\hat{c}^{2}}-A_{Z Z}\left(m_{Z}^{2}\right)\right]_{\overline{\mathrm{MS}}}+\frac{\alpha}{\pi} \frac{\hat{s}^{2}}{\hat{c}^{2}} d
\end{aligned}
$$

where $d$ and $\hat{d}$ are defined in Eqs. (9c) and (10d) and $A_{Z Z}\left(q^{2}\right)$ is the unrenormalized $Z Z$ transverse self-energy with the coupling $e^{2} / \hat{s}^{2}$ factored out [1]. Except for the terms involving $d$ and $\hat{d}$, which arise from the finite counterterms in Eqs. (7d) and (9a) associated with the decoupling of the top quark, Eq. (14) has the same form as Eq. (15b) of Ref. [1]. It is understood, however, that $\Delta \hat{r}_{W}, \hat{e}^{2}$, and $\hat{s}^{2}$ in Eq. (14) are defined according to the prescriptions of the present paper, namely Eqs. (10c), (8c), and (2). In Appendix B we show how Eqs. (10a)-(10c), and (14) can be derived from the results of Ref. [1] by neglecting very small contributions of $O\left(\alpha^{2}\right)$ without logarithmic or $m_{t}^{2} / m_{Z}^{2}$ enhancements, as well as terms of $O\left(\alpha^{3}\right)$. As pointed out in Ref. [1], if one neglects also subleading corrections of $O\left(\left(\alpha / \pi \hat{s}^{2}\right) x_{t}\right)$, with $x_{t}$ being defined in Eq. (17b), one can replace $\hat{e}^{2}\left[1-\Delta \hat{r}_{W}-(\alpha / \pi) \hat{d}\right] \rightarrow e^{2}$ in the second term of Eq. (14). As in Ref. [1], we have retained such subleading terms in Eq. (14) because the resulting expression describes very accurately the resummation of one-loop effects, which is particularly simple in the $\overline{\mathrm{MS}}$ framework.

We now turn our attention to the evaluation of the self-energies in the second term of Eq. (14). The bosonic contribution

$$
\left(e^{2} / \hat{s}^{2} m_{Z}^{2}\right) \operatorname{Re}\left[A_{W W}^{(b)}\left(m_{W}^{2}\right) / \hat{c}^{2}-A_{Z Z}^{(b)}\left(m_{Z}^{2}\right)\right]_{\overline{\mathrm{MS}}}
$$

is given in Eq. (A.9) of Ref. [1]. To study the fermionic contributions, we write

$$
C^{(f)} \equiv \frac{e^{2}}{\hat{s}^{2} m_{Z}^{2}} \operatorname{Re}\left[\frac{A_{W W}^{(f)}\left(m_{W}^{2}\right)}{\hat{c}^{2}}-A_{Z Z}^{(f)}\left(m_{Z}^{2}\right)\right]_{\overline{\mathrm{MS}}},
$$

and expand

$$
C^{(f)}=C_{0}^{(f)}+C_{\mathrm{QCD}}^{(f)}
$$

where $C_{0}^{(f)}$ and $C_{\mathrm{QCD}}^{(f)}$ are the $O(\alpha)$ and $O\left(\alpha \alpha_{s}\right)$ corrections, respectively. If the small terms proportional to $\alpha m_{q}^{2} / m_{W}^{2}(q=d, s, b)$ are neglected, the mixing angles are once more irrelevant and, to zeroth order in $\alpha_{s}$, we have (cf. Eq. (A.8) of Ref. [1]) 


$$
\begin{aligned}
C_{0}^{(f)}= & \frac{\alpha}{2 \pi \hat{s}^{2}} \frac{c^{2}}{\hat{c}^{2}}\left\{2\left(\ln c^{2}-\frac{5}{3}\right)+\frac{\ln \omega_{t}}{2}+\frac{\omega_{t}}{8}\left(1+2 \omega_{t}\right)+\frac{\left(\omega_{t}-1\right)^{2}}{2}\left(1+\omega_{t} / 2\right) \ln \left(1-\frac{1}{\omega_{t}}\right)\right\} \\
& +\frac{\alpha}{2 \pi \hat{s}^{2} \hat{c}^{2}}\left\{\frac{5}{3}\left(\frac{7}{4}-\frac{10}{3} \hat{s}^{2}+\frac{40}{9} \hat{s}^{4}\right)-\frac{1}{8}\left[1+\left(1-\frac{8}{3} \hat{s}^{2}\right)^{2}\right]\left[\ln \mu_{t}+\frac{1}{3}+2\left(1+2 \mu_{t}\right)\left(\Lambda\left(D_{t}\right)-1\right)\right]\right\} \\
& +\frac{3 \alpha}{4 \pi \hat{s}^{2} \hat{c}^{2}} \mu_{t}\left(\frac{1}{4}+\Lambda\left(D_{t}\right)-1\right),
\end{aligned}
$$

where $\mu_{t}=m_{t}^{2} / m_{Z}^{2}, D_{t}=4 \mu_{t}-1$, and $\Lambda(D)=D^{1 / 2} \tan ^{-1}\left(D^{-1 / 2}\right)$ for $D>0$. In analogy with our discussion of $B_{0}^{(f)}$, in Appendix $\mathrm{C}$ we give the expression for $C_{0}^{(f)}$ for arbitrary fermion masses in the approximation of neglecting the squares of mixing angles. We find that the corrections of $O\left(\mathrm{~m}^{2} / \mathrm{m}_{W}^{2}\right)$ to $C_{0}^{(f)}$ are also of order $10^{-5}$.

The contribution of $O\left(\alpha \alpha_{s}\right)$ in Eq. (15b) is given by

$$
\begin{gathered}
C_{\mathrm{QCD}}^{(f)}=\frac{\alpha}{4 \pi \hat{s}^{2} \hat{c}^{2}}\left\{\frac{2 \hat{\alpha}_{s}\left(m_{Z}\right)}{\pi}\left[c^{2} \ln c^{2}+\left(4 \zeta(3)-\frac{55}{12}\right)\left(-s^{2}+2 \hat{s}^{2}\left(1-\frac{10}{9} \hat{s}^{2}\right)\right)\right]\right. \\
+\frac{\hat{\alpha}_{s}\left(m_{t}\right)}{\pi}\left[c^{2} \ln c^{2}+\left(4 \zeta(3)-\frac{55}{12}\right)\left(-s^{2}+2 \hat{s}^{2}\left(1-\frac{10}{9} \hat{s}^{2}\right)\right)-c^{2}\left(4 \omega_{t} F_{1}\left(\frac{1}{\omega_{t}}\right)-\ln \omega_{t}\right)\right. \\
\left.\left.+\left(1-\frac{8}{3} \hat{s}^{2}\right)^{2}\left(\mu_{t} V_{1}\left(\frac{1}{4 \mu_{t}}\right)-\frac{\ln \mu_{t}}{4}\right)+\mu_{t} A_{1}\left(\frac{1}{4 \mu_{t}}\right)-\frac{\ln \mu_{t}}{4}\right]\right\},
\end{gathered}
$$

where the functions $F_{1}(x), V_{1}(r)$, and $A_{1}(r)$ are defined in Ref. [25].

The leading asymptotic behavior of $C_{0}^{(f)}$ for large $m_{t}$ is $\left(3 \alpha / 16 \pi \hat{s}^{2} \hat{c}^{2}\right)\left(m_{t}^{2} / m_{Z}^{2}\right)$, which arises from the last term of Eq. (15c). In the $O\left(\alpha \alpha_{s}\right)$ corrections the leading contribution is contained in the combination

$$
\left[\alpha \hat{\alpha}_{s}\left(m_{t}\right) / 4 \pi^{2} \hat{s}^{2} \hat{c}^{2}\right]\left\{\mu_{t} A_{1}\left[1 /\left(4 \mu_{t}\right)\right]-4 c^{2} \omega_{t} F_{1}\left(1 / \omega_{t}\right)\right\},
$$

which asymptotically becomes

$$
-\left[\alpha \hat{\alpha}_{s}\left(m_{t}\right) / 8 \pi^{2} \hat{s}^{2} \hat{c}^{2}\right]\left(\pi^{2} / 3+1\right)\left(m_{t}^{2} / m_{Z}^{2}\right) .
$$

Combining these contributions, inserting the result in Eq. (14) and neglecting there the subleading $O\left(\alpha^{2}\right)$ contributions, we have

$$
\Delta \hat{r} \sim-\frac{3 \alpha}{16 \pi \hat{s}^{2} \hat{c}^{2}} \frac{m_{t}^{2}}{m_{Z}^{2}}\left[1-\frac{2 \hat{\alpha}_{s}\left(m_{t}\right)}{3 \pi}\left(\frac{\pi^{2}}{3}+1\right)\right] \quad\left(m_{t} \gg m_{Z}\right),
$$

while the leading asymptotic behavior for large $m_{H}$ is the same as in Ref. [12], namely

$$
\Delta \hat{r} \sim \frac{\alpha}{2 \pi \hat{s}^{2} \hat{c}^{2}}\left(\frac{5}{6}-\frac{3}{4} \hat{c}^{2}\right) \ln \frac{m_{H}}{m_{Z}} \quad\left(m_{H} \gg m_{Z}\right) .
$$

The term involving $\hat{\alpha}_{s}$ in the last factor of Eq. (16a) represents the most important $O\left(\alpha \alpha_{s}\right)$ correction. Indeed, the contribution of Eq. (16a) depends very sensitively on $m_{t}$ and the coefficient of $\hat{\alpha}_{s} / \pi$ in the last factor, namely -2.86 , is quite large. The presence of this $\hat{\alpha}_{s}$ correction induces an increase in the value of $m_{t}$ read from experiments of approximately

$$
\frac{\Delta m_{t}}{m_{t}} \approx \frac{\hat{\alpha}_{s}\left(m_{t}\right)}{3 \pi}\left(\frac{\pi^{2}}{3}+1\right) \approx 0.455 \hat{\alpha}_{s}\left(m_{t}\right)
$$

which amounts to $\Delta m_{t} \approx(4.9,7.5,9.6,11.7) \mathrm{GeV}$ for $m_{t}=m_{Z}, 150,200,250 \mathrm{GeV}$. It should be stressed that, as is obvious from the structure of Eq. (16a), these results depend sensitively on the precise definition of $m_{t}$. The quantity that appears in Eq. (16a) and the various expressions of this paper is the zero of the real part of the in- verse propagator. In the literature it is variously referred to as the "physical," "on-shell," or "dressed" mass. In the approximation of neglecting the $s$ dependence of the imaginary part of the top-quark self-energy, it coincides with the real part of the complex pole position [13-15]. It is also the mass that occurs in the Balmer formula for the toponium levels in the nonrelativistic bound-state picture and the parameter that governs the start of the $t \bar{t}$ cut in perturbation theory [44]. All the recent calculations of $O\left(\alpha \alpha_{s}\right)$ contributions and $t \bar{t}$ production [22-31] employ this definition or slight modifications thereof. It is worthwhile to notice that the $O\left(\hat{\alpha}_{s}\right)$ corrections become much smaller if one employs other definitions of mass [45]. For example, $m_{t}$ and the running mass, $\hat{m}_{t}\left(\hat{m}_{t}\right)$, are related by [44]

$$
m_{t}=\hat{m}_{t}\left(\hat{m}_{t}\right)\left[1+4 \hat{\alpha}_{s} /(3 \pi)+O\left(\hat{\alpha}_{s}^{2}\right)\right] .
$$

Inserting this into Eq. (16a), we get a contribution involving

$$
\left[\hat{m}_{t}\left(\hat{m}_{t}\right)\right]^{2}\left[1-2\left(\hat{\alpha}_{s} / \pi\right)\left(\pi^{2} / 9-1\right)\right]
$$

and we see that the coefficient of $\hat{\alpha}_{s} / \pi$ has changed from -2.86 to -0.19 . Similarly, if one expresses the cor- 
rections in terms of the Georgi-Politzer mass, $M\left(-m^{2}\right)$ [46], which is gauge dependent and usually evaluated in the Landau gauge, the coefficient of $\hat{\alpha}_{s} / \pi$ becomes even smaller, namely +0.09 . Because the perturbative evaluation of the $(t, b)$-isodoublet loops involves high mass scales, of $O\left(m_{t}\right)$ or $O\left(m_{Z}\right)$, both the on-shell and $\hat{m}_{t}\left(\hat{m}_{t}\right)$ definitions are in principle suitable, although the former is the natural choice in the DR approach [23-25, $30,31]$. The relevant question, of course, is what mass parametrization is more adequate to describe the physical issues at hand, namely the production and detection of the top quark. In this connection it also appears that the on-shell mass is the most appropriate parameter because, in the propagation of $t$ and $\bar{t}$ between the "production" and "decay" vertices, configurations near the "mass shell" will be greatly enhanced kinematically (resonance effect). Another consideration, of a more practical nature, is that the $m_{t}$ parametrization of the radiative corrections should be consistent with the one employed in the calculation of $t \bar{t}$ production [27-29] and, as mentioned before, this is again the "pole" or "on-shell" definition.

Returning to the evaluation of $\Delta \hat{r}$, we must still consider the irreducible contributions of $O\left(\alpha^{2}\left(m_{t}^{2} / m_{W}^{2}\right)^{2}\right)$. As mentioned before, these can be gleaned from the twoloop irreducible corrections to the $\rho$ parameter. The very recent work of Ref. [7] leads to a significant change in the magnitude of these corrections. Indeed, these authors find for the leading high- $m_{t}$ contributions to the $\rho$ parameter an expansion of the form

$$
\frac{1}{\rho}=1-x_{t}\left[1+R\left(\frac{m_{H}}{m_{t}}\right) \frac{x_{t}}{3}\right] \equiv 1-\Delta \bar{\rho},
$$

where

$$
x_{t}=\frac{3 G_{\mu} m_{t}^{2}}{8 \pi^{2} \sqrt{2}}
$$

is the one-loop term [47] and $R$ is a negative function of $m_{H} / m_{t}$. When $m_{H} / m_{t}=0, R$ equals $19-2 \pi^{2} \approx$ -0.7392 , the result of Ref. [6], but as $m_{H} / m_{t}$ increases, $R$ rapidly becomes more negative, reaching a minimum of $\approx-11.8$ for $m_{H} / m_{t} \approx 5.8$. In current discussions $m_{H} / m_{t}$ ranges from $\approx 0.24$ (corresponding to $m_{H} \approx$ $60 \mathrm{GeV}$ and $m_{t} \approx 250 \mathrm{GeV}$ ) to $\approx 11$ (corresponding to $m_{H} \approx 1 \mathrm{TeV}$ and $\left.m_{t} \approx 91 \mathrm{GeV}\right)$. Noting that $R(0.24) \approx$ -3 and $R(11) \approx-10$, it is clear that, although Refs. [6] and [7] agree in the limit $m_{H} / m_{t}=0$, for realistic values of this ratio the results of Ref. [7] tell us that these corrections are considerably larger in magnitude.

Calling $\Delta \hat{r}^{(1)}$ the "one-loop" expression for $\Delta \hat{r}$ given in Eq. (14), we include the two-loop irreducible contributions by writing

$$
\Delta \hat{r}=\Delta \hat{r}^{(1)}-\frac{1}{3} R\left(\frac{m_{H}}{m_{t}}\right) x_{t}^{2} \frac{\left(1-\Delta \hat{r}^{(1)}\right)^{2}}{1-\Delta \hat{r}_{W}}
$$

The rationale is the following. In the $\overline{\mathrm{MS}}$ scheme the $\rho$ parameter is naturally identified with $\hat{\rho} \equiv c^{2} / \hat{c}^{2}$ and, from Eqs. (2) and (3), we see that (cf. Eqs. (17a) and (17b) of Ref. [1])

$$
\hat{\rho}=\frac{1-\Delta \hat{r}}{1-\Delta \hat{r}_{W}} .
$$

Neglecting very small terms of order $\left(R x_{t}^{2} / 3\right)^{2}$, one indeed verifies that when the second term in Eq. (18a) is inserted in Eq. (18b), it leads to an additional contribution of $-R\left(m_{H} / m_{t}\right) x_{t}^{2} / 3$ to $1 / \hat{\rho}$, in conformity with Eq. (17a).

We have given all the elements necessary to evaluate the basic radiative corrections $\Delta \hat{r}_{W}[\mathrm{cf}$. Eq. (10c)] and $\Delta \hat{r}$ [cf. Eqs. (14) and (18a)] including $O\left(\alpha \alpha_{s}\right)$ corrections. In conjunction with

$$
\hat{s}^{2}=\frac{1}{2}\left\{1-\left[1-\frac{4 A^{2}}{m_{Z}^{2}(1-\Delta \hat{r})}\right]^{1 / 2}\right\},
$$

which follows from Eq. (3), $\Delta \hat{r}$ can be employed to calculate $\hat{s}^{2} \equiv \sin ^{2} \hat{\theta}_{W}\left(m_{Z}\right)$ in terms of the accurately known quantities $G_{\mu}, m_{Z}$, and $\alpha$, as a function of $m_{t}$ and $m_{H}$. The parameter $s^{2} \equiv 1-m_{W}^{2} / m_{Z}^{2}$ can be computed from (cf. Eq. (19) of Ref. [1])

$$
s^{2}=\hat{s}^{2}\left(1-\frac{\hat{c}^{2}}{\hat{s}^{2}} \frac{\Delta \hat{r}_{W}-\Delta \hat{r}}{1-\Delta \hat{r}_{W}}\right)
$$

and $\Delta r$ (cf. Eq. (22) of Ref. [1]) from

$$
\Delta r=\Delta \hat{r}_{W}-\frac{\hat{c}^{2}}{\hat{s}^{2}} \frac{\Delta \hat{r}_{W}-\Delta \hat{r}}{1-\left(\hat{c}^{2} / \hat{s}^{2}\right)\left(\Delta \hat{r}_{W}-\Delta \hat{r}\right) /\left(1-\Delta \hat{r}_{W}\right)} .
$$

Alternatively, writing $\hat{\rho} \equiv 1 /(1-\Delta \hat{\rho})$ we have (cf. Eqs. (17a), (17b), and (20) of Ref. [1])

$$
\begin{aligned}
\Delta \hat{\rho} & =\frac{\Delta \hat{r}_{W}-\Delta \hat{r}}{1-\Delta \hat{r}}, \\
1-\Delta r & =\left(1+\frac{c^{2}}{s^{2}} \Delta \hat{\rho}\right)\left(1-\Delta \hat{r}_{W}\right) .
\end{aligned}
$$

Again, the $m_{W}-m_{Z}$ interdependence can be expressed in two equivalent forms (cf. Eqs. (24) and (25) of Ref. [1]),

$$
\begin{aligned}
& \frac{m_{W}^{2}}{m_{Z}^{2}}=\frac{1}{2}\left\{1+\left[1-\frac{4 A^{2}}{m_{Z}^{2}(1-\Delta r)}\right]^{1 / 2}\right\}, \\
& \frac{m_{W}^{2}}{m_{Z}^{2}}=\frac{\hat{\rho}}{2}\left\{1+\left[1-\frac{4 A^{2}}{m_{Z}^{2} \hat{\rho}\left(1-\Delta \hat{r}_{W}\right)}\right]^{1 / 2}\right\} .
\end{aligned}
$$

The $W$ mass can be evaluated from (19b) or (19f) or (19g). Equations (19a)-(19g) have the same structure as in Ref. [1] because they follow from the same basic relations, namely Eqs. (1)-(3). In this paper, however, the explicit evaluation of $\Delta \hat{r}_{W}$ and $\Delta \hat{r}$ via Eqs. (10c), (14), and (18a) is somewhat different because we have included the $O\left(\alpha \alpha_{s}\right)$ corrections, updated the contributions of $e^{2} \operatorname{Re}\left[\Pi_{\gamma \gamma}^{(5)}(0)-\Pi_{\gamma \gamma}^{(5)}\left(m_{Z}^{2}\right)\right]$ and the two-loop irreducible parts, and implemented the decoupling of the top quark. By inference the same holds true for $\Delta r$ and $\Delta \hat{\rho}$ when they are evaluated from $\Delta \hat{r}_{W}$ and $\Delta \hat{r}$ via Eqs. (19c) and $(19 \mathrm{~d})$, respectively. It should be observed, moreover, 
that, except for very small effects of $O\left(\alpha^{2}\right)$, i.e., of the same order as those we have neglected, the decoupling of the top quark should not affect physical observables such as the radiative correction $\Delta r$ and the predicted value of $m_{W}$.

As shown in Appendix $\mathrm{C}$, the corrections of $O\left(m_{f}^{2} / m_{W}^{2}\right)$ to $\Delta r$ are also very small. However, they are enhanced relative to those of $\Delta \hat{r}_{W}$ and $\Delta \hat{r}$, and for $m_{Z} \leq m_{t} \leq 250 \mathrm{GeV}$ they vary from $\approx-7 \times 10^{-5}$ to $\approx 8 \times 10^{-5}$.

\section{RESIDUAL PARTS OF $\Delta \hat{r}_{W}, \Delta \hat{r}$, AND $\Delta r$}

It is a simple matter to derive expressions relating $m_{W}$ and $m_{Z}$ to $G_{\mu}, \hat{s}^{2}, \hat{c}^{2}$, and $\hat{e}^{2}$ (rather than $e^{2}$ ). To see this, we write Eq. (2) in the form

$$
\hat{s}^{2}=\frac{\pi \alpha}{\sqrt{2} G_{\mu} m_{W}^{2}} \frac{1}{1-(\alpha / \pi) \Delta_{\gamma}-\left[\Delta \hat{r}_{W}-(\alpha / \pi) \Delta_{\gamma}\right]} .
$$

Factoring out $\left[1-(\alpha / \pi) \Delta_{\gamma}\right]$ and recalling Eq. (8c), we have

$$
\hat{s}^{2}=\frac{\pi \hat{\alpha}}{\sqrt{2} G_{\mu} m_{W}^{2}} \frac{1}{1-\left(\Delta \hat{r}_{W}\right)_{\mathrm{res}}},
$$

where

$$
\left(\Delta \hat{r}_{W}\right)_{\mathrm{res}}=\left(\Delta \hat{r}_{W}-\frac{\alpha}{\pi} \Delta_{\gamma}\right) \frac{\hat{e}^{2}}{e^{2}}
$$

The correction $\left(\Delta \hat{r}_{W}\right)_{\text {res }}$ represents the "residual part" of $\Delta \hat{r}_{W}$ after we have subtracted the large contribution $(\alpha / \pi) \Delta_{\gamma}$, evaluated with the coupling $\hat{e}^{2}$ rather than $e^{2}$. As $\left(\Delta \hat{r}_{W}\right)_{\text {res }} \ll \Delta \hat{r}_{W}$, we see that the dominant part of $\Delta \hat{r}_{W}$ can be absorbed by employing $\hat{\alpha}$ rather than $\alpha$ as the zeroth-order coupling.

Starting with Eq. (3), the analogous argument leads to

$$
\hat{s}^{2} \hat{c}^{2}=\frac{\pi \hat{\alpha}}{\sqrt{2} G_{\mu} m_{Z}^{2}} \frac{1}{1-(\Delta \hat{r})_{\mathrm{res}}},
$$

where

$$
(\Delta \hat{r})_{\mathrm{res}}=\left(\Delta \hat{r}-\frac{\alpha}{\pi} \Delta_{\gamma}\right) \frac{\hat{e}^{2}}{e^{2}}
$$

Again $(\Delta \hat{r})_{\text {res }}$ is the "residual part" of $\Delta \hat{r}$. In connection with the inclusion of the two-loop irreducible contributions of $O\left(\alpha^{2}\left(m_{t}^{2} / m_{W}^{2}\right)^{2}\right)$ [cf. Eq. (18a)], it is easy to see that the "one-loop" $\left(\Delta \hat{r}^{(1)}\right)_{\text {res }}$ can be obtained by replacing $\Delta \hat{r}_{W} \rightarrow\left(\Delta \hat{r}_{W}\right)_{\text {res }},(\alpha / \pi) \hat{d} \rightarrow(\hat{\alpha} / \pi) \hat{d}$, and $(\alpha / \pi) d \rightarrow(\hat{\alpha} / \pi) d$ on the rhs of Eq. (14), and the final $(\Delta \hat{r})_{\text {res }}$ follows by substituting $\Delta \hat{r}^{(1)} \rightarrow\left(\Delta \hat{r}^{(1)}\right)$ res and $\Delta \hat{r}_{W} \rightarrow\left(\Delta \hat{r}_{W}\right)_{\text {res }}$ on the rhs of Eq. (18a). Of course, once $\Delta \hat{r}$ is known, one can directly use Eq. (21b) for the numerical evaluation of $(\Delta \hat{r})_{\text {res }}$.

The corresponding expression from Eq. (1) is

$$
s^{2}=\frac{\pi \hat{\alpha}}{\sqrt{2} G_{\mu} m_{W}^{2}} \frac{1}{1-(\Delta r)_{\mathrm{res}}},
$$

where

$$
(\Delta r)_{\mathrm{res}}=\left(\Delta r-\frac{\alpha}{\pi} \Delta_{\gamma}\right) \frac{\hat{e}^{2}}{e^{2}}
$$

is the residual part of $\Delta r$. One readily finds that this quantity can be obtained by simply substituting $\Delta \hat{r}_{W} \rightarrow$ $\left(\Delta \hat{r}_{W}\right)_{\text {res }}$ and $\Delta \hat{r} \rightarrow(\Delta \hat{r})_{\text {res }}$ everywhere on the rhs of Eq. (19c). Of course, Eq. (22b) can be directly used for numerical evaluations.

As illustrations, for $m_{H}=250 \mathrm{GeV}$ and $m_{t}=$ $150 \mathrm{GeV}$ we have $\Delta \hat{r}_{W}=7.02 \times 10^{-2}, \Delta \hat{r}=6.34 \times 10^{-2}$, and $\Delta r=4.74 \times 10^{-2}$, while $\left(\Delta \hat{r}_{W}\right)_{\text {res }}=3.6 \times 10^{-3}$, $(\Delta \hat{r})_{\mathrm{res}}=-3.6 \times 10^{-3}$, and $(\Delta r)_{\mathrm{res}}=-2.08 \times 10^{-2}$. The corresponding values for $m_{H}=250 \mathrm{GeV}$ and $m_{t}=$ $200 \mathrm{GeV}$ are $\Delta \hat{r}_{W}=7.08 \times 10^{-2}, \Delta \hat{r}=5.88 \times 10^{-2}$, and $\Delta r=2.90 \times 10^{-2}$, while $\left(\Delta \hat{r}_{W}\right)_{\text {res }}=4.3 \times 10^{-3}$, $(\Delta \hat{r})_{\text {res }}=-8.5 \times 10^{-3}$, and $(\Delta r)_{\text {res }}=-4.05 \times 10^{-2}$. Unlike $\left(\Delta \hat{r}_{W}\right)_{\text {res }}$ or $(\Delta \hat{r})_{\text {res }},(\Delta r)_{\text {res }}$ is quite large for $m_{t} \approx 200 \mathrm{GeV}$.

A correction similar to $(\Delta r)_{\text {res }}$ has been recently employed in Ref. [20]. The two quantities are, however, not identical because $\hat{\alpha}$, defined in the $\overline{\mathrm{MS}}$ scheme, differs somewhat from the effective parameter $\alpha\left(m_{Z}\right)=$ $(128.8)^{-1}$ used in that work. This illustrates the rather obvious but important fact that running couplings are scheme dependent.

\section{V. $\Delta r$ IN THE ON-SHELL SCHEME}

In this section we discuss the incorporation of the perturbative $O\left(\alpha \alpha_{s}\right)$ corrections to $\Delta r[2,3]$ in the on-shell scheme of renormalization [2]. We follow the formulation proposed recently in Ref. [8], based on the expression

$$
\Delta r=\Delta \alpha-\frac{c^{2}}{s^{2}} \Delta \bar{\rho}(1-\Delta \alpha)+(\Delta r)_{\mathrm{rem}},
$$

where $\Delta \alpha=0.0597 \pm 0.0009$ represents the contribution of the charged leptons and the first five quark flavors to the photon vacuum-polarization function evaluated at $q^{2}=m_{Z}^{2}$, i.e., $e^{2} \operatorname{Re}\left[\Pi_{\gamma \gamma}(0)-\Pi_{\gamma \gamma}\left(m_{Z}^{2}\right)\right]$,

$$
\Delta \bar{\rho}=x_{t}\left[1+R\left(\frac{m_{H}}{m_{t}}\right) \frac{x_{t}}{3}\right],
$$

$x_{t}$ is defined in Eq. (17b), and $R\left(m_{H} / m_{t}\right)$ [7] is the function discussed after Eq. (17a). The result for $\Delta \alpha$ quoted above includes the recent calculation of the first five quark flavor contributions [10] and, for this reason, it slightly differs from the central value of 0.0602 employed in Ref. [8]. The second term in Eq. (23a) involves the leading $m_{t}$-dependent correction $\Delta \bar{\rho}$ to $1-1 / \rho$ [cf. Eq. (17a)] and we see that, in the case of $\Delta r$, it is enhanced by a factor $c^{2} / s^{2}$. Its importance for large $m_{t}$ in the $m_{W}-m_{Z}$ interdependence was pointed out in 1980 , in the work of Marciano and one of us (A.S.) [41]. Since that time, this potential effect has been discussed by several authors $[4,5,22-26,48,49]$.

The separation into leading contributions [the first two terms in Eq. (23a)] and a "remainder" $(\Delta r)_{\text {rem }}$ is the same as was proposed in Refs. [4, 5, 26], except that we have included the recent results of Ref. [7] on the two- 
loop contribution to $\Delta \bar{\rho}$. It is important to note that $(\Delta r)_{\text {rem }}$ differs from the quantity $(\Delta r)_{\text {res }}$ introduced at the end of Sec. III. Whereas in the latter we subtract the large logarithmic corrections, in $(\Delta r)_{\text {rem }}$ we also exclude the leading $m_{t}$-dependent contributions. The formulation of Ref. [8] provides also a very specific prescription to calculate $(\Delta r)_{\text {rem }}$, namely

$$
\begin{aligned}
(\Delta r)_{\mathrm{rem}}= & \Delta r^{(1)}-\Delta \alpha+\frac{c^{2}}{s^{2}} X \\
& +\frac{c^{2}}{s^{2}}\left(\tilde{x}_{t}-X\right) \frac{\sqrt{2} G_{\mu} m_{W}^{2}(1-\Delta \alpha) s^{2}}{\pi \alpha}
\end{aligned}
$$

where $\Delta r^{(1)}$ is the familiar one-loop expression of Ref. [2], expressed in terms of $\alpha$ and $\alpha / s^{2}$ as expansion parameters,

$$
\begin{aligned}
& \tilde{x}_{t}=\frac{3 \alpha}{16 \pi s^{2}} \frac{m_{t}^{2}}{m_{W}^{2}} \\
& X=\frac{e^{2}}{s^{2}} \operatorname{Re}\left[\frac{A_{W W}\left(m_{W}^{2}\right)}{m_{W}^{2}}-\frac{A_{Z Z}\left(m_{Z}^{2}\right)}{m_{Z}^{2}}\right]_{\mathrm{fin}},
\end{aligned}
$$

and the subscript "fin" means "finite part," i.e., that the pole terms have been subtracted and $\mu^{\prime}$ has been set equal to $m_{Z}$. We also note that $X$ is a gauge-invariant quantity. In Eq. (24c) we follow the notation of Ref. [1], which differs from that of Refs. $[2,41]$ in that an explicit coupling $e^{2} / s^{2}$ has been factored out in the $A_{W W}$ and $A_{Z Z}$ self-energies. The term $-\Delta \alpha$ in Eq. (24a) subtracts from $\Delta r^{(1)}$ the large logarithmic corrections which are included as part of the leading contributions in Eq. (23a). The quantity $\left(c^{2} / s^{2}\right) X$ subtracts another important part of $\Delta r^{(1)}$, which is then treated more accurately in the second term of Eq. (23a) and the last term of Eq. (24a), according to the following prescription. Decomposing $-\left(c^{2} / s^{2}\right) X=-\left(c^{2} / s^{2}\right) \tilde{x}_{t}+\left(c^{2} / s^{2}\right)\left(\tilde{x}_{t}-X\right)$, the dominant part, $-\left(c^{2} / s^{2}\right) \tilde{x}_{t}$, is included in the second term of Eq. (23a) with the effective coupling constant changed according to $\alpha / s^{2} \rightarrow \sqrt{2} G_{\mu} m_{W}^{2}(1-\Delta \alpha) / \pi$. The nondominant part, $\left(c^{2} / s^{2}\right)\left(\tilde{x}_{t}-X\right)$, is treated with the same coupling modification but it is retained as part of $(\Delta r)_{\text {rem }}$ [the last term of Eq. (24a)]. The rationale for this treatment of $\left(c^{2} / s^{2}\right)\left(\tilde{x}_{t}-X\right)$ was explained in Refs. [8, 50] and reflects the fact that a careful analysis of the resummation of one-loop effects leads to an expression of the form of Eq. (23a) in which $X$, rather than its dominant part $x_{t}$, is multiplied by $\sqrt{2} G_{\mu} s^{2} m_{W}^{2}(1-\Delta \alpha) /(\pi \alpha)=$ $(1-\Delta \alpha) /(1-\Delta r)$. This is natural because, since $\Delta r$ is the radiative correction in the relation between $m_{W}, m_{Z}, G_{\mu}$, and $\alpha$, it should involve quantities evaluated at $q^{2}=m_{Z}^{2}$ and $q^{2}=m_{W}^{2}$ such as $X$, rather than amplitudes evaluated at $q^{2}=0$ such as $\Delta \bar{\rho}$. We also point out that the neglect of this effect in Eq. (24a), namely the replacement $\sqrt{2} G_{\mu} m_{W}^{2} s^{2}(1-\Delta \alpha) /(\pi \alpha) \rightarrow 1$ in the last term, would induce a change $\approx\left(c^{2} / s^{2}\right)\left(\tilde{x}_{t}-X\right)\left(c^{2} / s^{2}\right) \Delta \bar{\rho}=$ $O\left(\left(\alpha / \pi s^{2}\right)\left(c^{2} / s^{2}\right)^{2} \Delta \bar{\rho}\right)$; although formally subleading, this is enhanced by two powers of $c^{2} / s^{2}$ and is, therefore, significantly larger than the expected theoretical error. It was already pointed out in Ref. [8] that Eqs. (23a) and (24a) include correctly not only the leading terms of $O\left(\alpha^{2}\left(m_{t}^{2} / m_{W}^{2}\right)^{2}\right)$ and $O\left(\alpha^{2} \ln ^{2}\left(m_{Z} / m_{f}\right)\right)$, where $m_{f}$ is a generic fermion mass, but also the subleading contributions of $O\left(\alpha^{2} \ln \left(m_{Z} / m_{f}\right)\right)$.

We now turn our attention to the incorporation of the perturbative $O\left(\alpha \alpha_{s}\right)$ contributions. We first consider Eq. (24a). The amplitudes modified by the QCD corrections are $X^{(f)}$ in the last term and the self-energy contributions $\left(e^{2} / s^{2} m_{W}^{2}\right) \operatorname{Re}\left[A_{W W}^{(f)}\left(m_{W}^{2}\right)-A_{W W}^{(f)}(0)\right]_{\text {fin }}$ and $e^{2}\left[\Pi_{\gamma \gamma}^{(f)}(0)\right]_{\text {fin }}$ contained in $\Delta r^{(1)}$, where the superscript $(f)$ denotes again fermionic contributions. The first two are obtained from $C^{(f)}$ [cf. Eqs. (15a)-(15d)] and $B^{(f)}[\mathrm{cf}$. Eqs. (11a)-(11d)], respectively, by simply changing everywhere $\hat{s}^{2} \rightarrow s^{2}$ and $\hat{c}^{2} \rightarrow c^{2}$. In particular, their QCD corrections are derived from Eqs. (15d) and (11d), respectively. The correction $e^{2}\left[\Pi_{\gamma \gamma}^{(f)}(0)\right]_{\text {fin }}$ can be read from Eq. (7a) by removing the pole terms, setting $\mu^{\prime}=m_{Z}$, and substituting $\hat{\alpha} \rightarrow \alpha$. It is not necessary to consider $\left(c^{2} / s^{2}\right) X^{(f)}$ in the third term of Eq. (24a) because, as explained before, it cancels an identical contribution in $\Delta r^{(1)}$. The value of $\Delta \alpha$ is not modified, as QCD corrections have already been taken into account in its evaluation.

In the above discussion, the quantities $\tilde{x}_{t}$ in Eq. (24a) and $\Delta \bar{\rho}$ in Eq. (23a) have not been altered, so that, except for $\Delta \alpha$, all the QCD corrections are contained in $(\Delta r)_{\mathrm{rem}}$. We may wish, however, to incorporate the leading QCD corrections in the second term of Eq. (23a). To achieve this, we subtract them from Eq. (24a) by replacing

$\tilde{x}_{t} \rightarrow \tilde{x}_{t}^{\prime}=\frac{3 \alpha}{16 \pi s^{2}} \frac{m_{t}^{2}}{m_{W}^{2}}\left[1-\frac{2 \hat{\alpha}_{s}\left(m_{t}\right)}{3 \pi}\left(\frac{\pi^{2}}{3}+1\right)\right]$

in the last term of that equation and, at the same time, we substitute

$$
\begin{aligned}
\Delta \bar{\rho} & \rightarrow \Delta \bar{\rho}^{\prime} \\
& =x_{t}\left[1-\frac{2 \hat{\alpha}_{s}\left(m_{t}\right)}{3 \pi}\left(\frac{\pi^{2}}{3}+1\right)+R\left(\frac{m_{H}}{m_{t}}\right) \frac{x_{t}}{3}\right]
\end{aligned}
$$

in Eq. (23a). The overall evaluation of $\Delta r$ is, of course, the same whether we employ $\Delta \bar{\rho}$ in Eq. (23a) and $\tilde{x}_{t}$ in Eq. (24a) or the modified quantities, $\Delta \bar{\rho}^{\prime}$ and $\tilde{x}_{t}^{\prime}$. In the second formulation, however, the first two terms of Eq. (23a), with $\Delta \bar{\rho} \rightarrow \Delta \bar{\rho}^{\prime}$, describe more accurately the leading $m_{t}$-dependent corrections.

The contributions of $O\left(\alpha^{2} \hat{\alpha}_{s}, \alpha \hat{\alpha}_{s}^{2}\right)$ are unknown at the present time and for this reason we have not made any attempt to include them. However, the structure of Eq. (25b) gives a hint about what their magnitude might be. Suppose, for example, that the leading QCD effects are always very small when the electroweak corrections are expressed in terms of the running mass, $\hat{m}_{t}\left(\hat{m}_{t}\right)$, as it happens with the $O\left(\alpha \hat{\alpha}_{s}\right)$ corrections. In that hypothetical case, the discussion after Eq. (16c) indicates that the modified $\Delta \bar{\rho}$ parameter would be obtained approximately by appending a factor $\left\{1-\left[2 \hat{\alpha}_{s}\left(m_{t}\right) / 3 \pi\right]\left(\pi^{2} / 3+1\right)\right\}$ to each $x_{t}$ in Eq. (23b). For $m_{H}=600 \mathrm{GeV}$ and $m_{t}=200 \mathrm{GeV}$, the difference with Eq. (25b) would lead to an aditional contribution to $\Delta r$ of $-3.4 \times 10^{-4}$. This is of the same order of magnitude as the sublead- 
ing terms of $O\left(\left(\alpha / \pi s^{2}\right)\left(c^{2} / s^{2}\right) x_{t}\right)$, discussed in Sec. I. The surprisingly large size of these possible corrections of $O\left(\left(c^{2} / s^{2}\right) \hat{\alpha}_{s} x_{t}^{2}\right)$ is due to the $m_{t}^{4}$ dependence and the considerable magnitude of the function $R$. This observation illustrates the fact that, for large $m_{t}$ values, the theoretical error due to unknown higher-order corrections may arise from very different sources.

\section{THRESHOLD EFFECTS}

The fermionic contribution to the vacuum polarization of the intermediate vector bosons can be expressed in terms of the amplitudes

$$
\begin{aligned}
& \Pi_{\mu \nu}^{V, A}\left(q, m_{1}, m_{2}\right) \\
& \quad=-i \int d^{4} x e^{i q \cdot x}\left\langle 0\left|T^{*}\left[J_{\mu}^{V, A}(x) J_{\nu}^{V, A \dagger}(0)\right]\right| 0\right\rangle,
\end{aligned}
$$

where $T^{*}$ is the covariant time-ordered product and $J_{\mu}^{V}=\bar{\psi}_{1} \gamma_{\mu} \psi_{2}$ and $J_{\mu}^{A}=\bar{\psi}_{1} \gamma_{\mu} \gamma_{5} \psi_{2}$ are the vector and axial-vector currents, constructed with the spinors fields $\psi_{1}$ and $\psi_{2}$ endowed with masses $m_{1}$ and $m_{2}$, respectively. Thus, except for vector currents with $m_{1}=m_{2}$, the conservation of the currents is explicitly broken by mass terms. In turn, the tensors $\Pi_{\mu \nu}^{V, A}$ have the well-known structure

$$
\begin{aligned}
\Pi_{\mu \nu}^{V, A}\left(q, m_{1}, m_{2}\right)= & \Pi^{V, A}\left(s, m_{1}, m_{2}\right) g_{\mu \nu} \\
& +\lambda^{V, A}\left(s, m_{1}, m_{2}\right) q_{\mu} q_{\nu},
\end{aligned}
$$

where, throughout this section, $s \equiv q^{2}$.

Threshold effects involving the $t \bar{t}, t \bar{b}$, and $b \bar{b}$ channels can be expressed as contributions to the imaginary parts of the functions $\Pi^{V, A}$ and $\lambda^{V, A}[24,27-31]$. A number of papers $[23,24]$ have made use of DR's to express the physically important amplitudes $\Pi^{V, A}$ in terms of their imaginary parts. In Refs. $[30,31]$ two of us (B.K. and A.S.) proposed to use DR's directly constructed from the Ward identities. We recall the basic strategy: contracting both sides of Eq. (26a) with $q^{\mu}$, one derives the relation

$$
\begin{aligned}
\Pi^{V, A}\left(s, m_{1}, m_{2}\right)= & -s \lambda^{V, A}\left(s, m_{1}, m_{2}\right) \\
& +\Delta^{V, A}\left(s, m_{1}, m_{2}\right),
\end{aligned}
$$

where $\Delta^{V, A}\left(s, m_{1}, m_{2}\right)$ is defined by

$$
\begin{aligned}
\int d^{4} x e^{i q \cdot x}\langle 0| T\left[\partial^{\mu} J_{\mu}^{V, A}(x)\right. & \left.J_{\nu}^{V, A \dagger}(0)\right]|0\rangle \\
& \equiv \Delta^{V, A}\left(s, m_{1}, m_{2}\right) q_{\nu} .
\end{aligned}
$$

The idea then is to write DR's for $\lambda^{V, A}$ and $\Delta^{V, A}$ and to obtain $\Pi^{V, A}$ by means of Eq. (27a). This approach has been employed in Refs. $[30,31]$ to discuss both the perturbative and the threshold contributions in the onshell scheme of renormalization. The aim of this section is to extend the discussion, so that the threshold effects can also be included in the $\overline{\mathrm{MS}}$ calculations.

We recall that in our analysis the threshold contributions to the imaginary parts are nonzero over a small, finite range, i.e., they have compact support. As a consequence, the corresponding unsubtracted DR integrals for $\lambda^{V, A}$ and $\Delta^{V, A}$ are convergent and, moreover, vanish as $|s| \rightarrow \infty$. In using the DR approach, we selfconsistently assume that the threshold contributions to $\lambda^{V, A}$ and $\Delta^{V, A}$ tend to zero as $|s| \rightarrow \infty$, so that these quantities satisfy unsubtracted DR's. Thus,

$$
\begin{aligned}
\lambda^{V, A}\left(s, m_{1}, m_{2}\right) & =\frac{1}{\pi} \int d s^{\prime} \frac{\operatorname{Im} \lambda^{V, A}\left(s^{\prime}, m_{1}, m_{2}\right)}{s^{\prime}-s-i \epsilon} \\
\Delta^{V, A}\left(s, m_{1}, m_{2}\right) & =\frac{1}{\pi} \int d s^{\prime} \frac{\operatorname{Im} \Delta^{V, A}\left(s^{\prime}, m_{1}, m_{2}\right)}{s^{\prime}-s-i \epsilon} .
\end{aligned}
$$

Inserting these expressions into Eq. (27a) and using the same equation to relate the imaginary parts, one obtains the two equivalent representations [31]

$$
\begin{aligned}
\Pi^{V, A}\left(s, m_{1}, m_{2}\right)=\frac{1}{\pi} \int d s^{\prime}\left[\frac{\operatorname{Im} \Pi^{V, A}\left(s^{\prime}, m_{1}, m_{2}\right)}{s^{\prime}-s-i \epsilon}\right. & \left.+\operatorname{Im} \lambda^{V, A}\left(s^{\prime}, m_{1}, m_{2}\right)\right], \\
\Pi^{V, A}\left(s, m_{1}, m_{2}\right)= & \frac{s}{\pi} \int \frac{d s^{\prime}}{s^{\prime}} \frac{\operatorname{Im} \Pi^{V, A}\left(s^{\prime}, m_{1}, m_{2}\right)}{s^{\prime}-s-i \epsilon} \\
& +\frac{1}{\pi} \int \frac{d s^{\prime}}{s^{\prime}} \operatorname{Im} \Delta^{V, A}\left(s^{\prime}, m_{1}, m_{2}\right) .
\end{aligned}
$$

In Eqs. (28a) $-(29 \mathrm{~b})$ and henceforth it is understood that $\lambda^{V, A}, \Delta^{V, A}$, and $\Pi^{V, A}$ represent the excess threshold contributions relative to the perturbative $O\left(\alpha \alpha_{s}\right)$ corrections. As explained in Ref. [31], Eq. (29b) can be directly derived from the following assumptions: (i) $\Pi^{V, A}$ satisfies a once-subtracted DR; ( $i i)$ the subtraction constant is determined from the Ward identity (27a), so that $\Pi^{V, A}(0)=\Delta^{V, A}(0) ;($ iii $) \Delta^{V, A}(s)$ satisfies an unsubtracted DR, so that $\Delta^{V, A}(0)$ can be calculated from the second integral in Eq. (29b). The fact that $\Pi^{V, A}$ must satisfy a subtracted DR can be clearly seen by considering the particular case of vector currents with equal masses. In that case $\lambda^{V}\left(s^{\prime}, m, m\right)=-\Pi^{V}\left(s^{\prime}, m, m\right) / s^{\prime}$ and $\Delta^{V}\left(s^{\prime}, m, m\right)=0$, so that Eqs. (29a) and (29b) reduce to

$$
\Pi^{V}(s, m, m)=\frac{s}{\pi} \int \frac{d s^{\prime}}{s^{\prime}} \frac{\operatorname{Im} \Pi^{V}\left(s^{\prime}, m, m\right)}{s^{\prime}-s-i \epsilon},
$$

which vanishes at $s=0$, in conformity with the Ward identity (27a). If, instead, $\Pi^{V}(s, m, m)$ were to satisfy an unsubtracted DR, the condition $\Pi^{V}(0, m, m)=0$ would imply $\int d s^{\prime} \operatorname{Im} \Pi^{V}\left(s^{\prime}, m, m\right) / s^{\prime}=0$, which is manifestly false, as $\operatorname{Im} \Pi^{V}\left(s^{\prime}, m, m\right) \geq 0$. In summary, Eqs. (29a) and $(29 \mathrm{~b})$ are the simplest possible DR's consistent with the Ward identity (27a). As mentioned before, the latter is a crucial requirement. We also see from Eq. (29a) that the threshold effects modify the asymptotic behavior of the full $\Pi^{V, A}(s)$ as $|s| \rightarrow \infty$ by constants, i.e., by subleading terms. [We recall that the perturbative contributions to $\Pi^{V, A}(s)$ grow as $s$, modulo logarithms.]

Threshold effects associated with the $t \bar{b}$ channels are 
greatly suppressed because they are proportional to the squared reduced mass of the quarks [24] and can be neglected. Those involving the $b \bar{b}$ channel, i.e., "bottomium resonances," give significant contributions only to $e^{2} \Pi_{\gamma \gamma}(0)$ and are already included in the evaluation of $e^{2} \operatorname{Re}\left[\Pi_{\gamma \gamma}^{(5)}(0)-\Pi_{\gamma \gamma}^{(5)}\left(m_{Z}^{2}\right)\right]$. Thus, only the case $m_{1}=m_{2}=m_{t}$ is significant. For vector currents with equal masses, the relevant DR is given in Eq. (30a). For axial-vector currents, we employ

$$
\begin{aligned}
\Pi^{A}(s, m, m)=\frac{1}{\pi} \int d s^{\prime} & {\left[\frac{\operatorname{Im} \Pi^{A}\left(s^{\prime}, m, m\right)}{s^{\prime}-s-i \epsilon}\right.} \\
& \left.+\operatorname{Im} \lambda^{A}\left(s^{\prime}, m, m\right)\right],
\end{aligned}
$$

in accordance with Eq. (29a). The amplitude $\operatorname{Im} \Pi^{A}\left(s^{\prime}, m_{t}, m_{t}\right)$ receives contributions from $J^{P}=1^{+}$ states, i.e., $l=1$, which are suppressed near threshold by centrifugal barrier effects. On the other hand, $\operatorname{Im} \lambda^{A}\left(s^{\prime}, m_{t}, m_{t}\right)$ also receives significant contributions from $0^{-}$states, i.e., $l=0$. A detailed discussion of $\operatorname{Im} \Pi^{V}\left(s^{\prime}, m_{t}, m_{t}\right)$ and $\operatorname{Im} \lambda^{A}\left(s^{\prime}, m_{t}, m_{t}\right)$ in both the resonance [24] and Green-function [28] approaches is given in Ref. [31]. In both cases one finds [31]

$$
\begin{aligned}
\operatorname{Im} \lambda^{A}\left(s^{\prime}, m_{t}, m_{t}\right) & \approx \operatorname{Im} \lambda^{V}\left(s^{\prime}, m_{t}, m_{t}\right) \\
& =-\frac{\operatorname{Im} \Pi^{V}\left(s^{\prime}, m_{t}, m_{t}\right)}{s^{\prime}} .
\end{aligned}
$$

The second equality is, of course, an exact consequence of the Ward identity (27a).

We now discuss the specific threshold contributions to the basic radiative corrections studied in the paper. The corrections $\Delta_{\gamma}$ and $\Delta \hat{r}_{W}$ contain $e^{2} \Pi_{\gamma \gamma}^{(f)}(0)$ [cf. Eqs. (10a) and $(10 \mathrm{~b})]$. The top contribution to this amplitude is obtained from Eq. (A7),

$$
e^{2} \Pi_{\gamma \gamma}^{(t)}(0)=e^{2} \frac{4}{9}\left(\Pi^{V}\right)^{\prime}\left(0, m_{t}, m_{t}\right),
$$

where $\left(\Pi^{V}\right)^{\prime}\left(0, m_{t}, m_{t}\right)=\left.(\partial / \partial s) \Pi^{V}\left(s, m_{t}, m_{t}\right)\right|_{s=0}$. Recalling Eq. (30a), we have

$$
\left(\Pi^{V}\right)^{\prime}\left(0, m_{t}, m_{t}\right)=\frac{1}{\pi} \int \frac{d s^{\prime}}{{s^{\prime}}^{2}} \operatorname{Im} \Pi^{V}\left(s^{\prime}, m_{t}, m_{t}\right) .
$$

There are no additional significant threshold effects in $\Delta \hat{r}_{W}$, because those involving $\operatorname{Re}\left[A_{W W}\left(m_{W}^{2}\right)-\right.$ $\left.A_{W W}(0)\right] / m_{W}^{2}$ [cf. Eq. (10a)] are suppressed by reducedmass effects. In the case of $\Delta \hat{r}$ [cf. Eq. (14)] the same holds true for the term involving $\operatorname{Re} A_{W W}\left(m_{W}^{2}\right) /\left(\hat{c}^{2} m_{Z}^{2}\right)$; there are, however, significant threshold contributions to $\operatorname{Re} A_{Z Z}\left(m_{Z}^{2}\right) / m_{Z}^{2}$. According to Eq. (A13)

$$
\begin{aligned}
& \frac{\operatorname{Re} A_{Z Z}^{(t)}\left(m_{Z}^{2}\right)}{m_{Z}^{2}} \\
& =-\frac{1}{16 \hat{c}^{2} m_{Z}^{2}} \operatorname{Re}\left[\left(1-\frac{8}{3} \hat{s}^{2}\right)^{2} \Pi^{V}\left(m_{Z}^{2}, m_{t}, m_{t}\right)\right. \\
& \left.+\Pi^{A}\left(m_{Z}^{2}, m_{t}, m_{t}\right)\right] .
\end{aligned}
$$

Using Eqs. (30a)-(30c), we have

$$
\begin{aligned}
\frac{\operatorname{Re} \Pi^{V}\left(m_{Z}^{2}, m_{t}, m_{t}\right)}{m_{Z}^{2}} & =\frac{1}{\pi} \mathcal{P} \int \frac{d s^{\prime}}{s^{\prime}} \frac{\operatorname{Im} \Pi^{V}\left(s^{\prime}, m_{t}, m_{t}\right)}{s^{\prime}-m_{Z}^{2}}, \\
\frac{\operatorname{Re} \Pi^{A}\left(m_{Z}^{2}, m_{t}, m_{t}\right)}{m_{Z}^{2}} & \approx \frac{1}{\pi m_{Z}^{2}} \int d s^{\prime} \operatorname{Im} \lambda^{A}\left(s^{\prime}, m_{t}, m_{t}\right) \\
& \approx-\frac{1}{\pi m_{Z}^{2}} \int \frac{d s^{\prime}}{s^{\prime}} \operatorname{Im} \Pi^{V}\left(s^{\prime}, m_{t}, m_{t}\right),
\end{aligned}
$$

where $\mathcal{P}$ denotes the principal value of the integral. We note that, as explained earlier, the threshold behavior of $\operatorname{Im} \Pi^{A}\left(s^{\prime}, m_{t}, m_{t}\right)$ is suppressed by centrifugal barrier effects and its contribution has been neglected in Eq. (32c). As the support for the threshold contributions to $\operatorname{Im} \Pi^{V}\left(s^{\prime}, m_{t}, m_{t}\right)$ is located in the neighborhood of $s^{\prime} \approx 4 m_{t}^{2}$, the dominant effect for $m_{t}^{2} \gg m_{Z}^{2}$ is given by Eq. (32c), with Eqs. (31b) and (32b) being relatively suppressed by a factor $m_{Z}^{2} /\left(4 m_{t}^{2}\right)$. Furthermore, the term involving $\operatorname{Re}^{V}\left(m_{Z}^{2}, m_{t}, m_{t}\right)$ in Eq. (32a) has a small cofactor $\left(1-8 \hat{s}^{2} / 3\right)^{2} \approx 0.14$. In the range $m_{Z} \leq m_{t} \leq$ $250 \mathrm{GeV}, \mathrm{Eq}$. (32c) increases more rapidly than linearly with $m_{t}$, while the contributions of Eqs. (31b) and (32b) to $\Delta \hat{r}$ and $\Delta r$ remain very small, at most a few times $10^{-5}$. As discussed in Ref. [31], the sign of Eq. (32c) can be understood with an argument reminiscent of the one employed in technicolor theories to explain the generation of the vector-boson masses: $0^{-}$states contribute to the functions $\lambda^{A}(s)$ and $\Delta^{A}(s)$ and, via the Ward identity (27a), to $\Pi^{A}(s)$. In technicolor theories this gives rise to $m_{Z}^{2} \sim A_{Z Z}(0)$. In our case, when Eq. (32c) is inserted in Eq. (32a), it leads to a positive contribution to the $Z^{0}$ mass shift, $\delta m_{Z}^{2}=\operatorname{Re} A_{Z Z}\left(m_{Z}^{2}\right)$.

Inserting into Eqs. (31b), (32b), and (32c) the detailed evaluations of $\operatorname{Im} \Pi^{V}\left(s^{\prime}, m_{t}, m_{t}\right)$ and $\operatorname{Im} \lambda^{A}\left(s^{\prime}, m_{t}, m_{t}\right)$ [31] derived from the analyses of Refs. [24,28], we obtain the additional contributions to $\Delta \hat{r}_{W}$ and $\Delta \hat{r}$ associated with the $t \bar{t}$ threshold. Because the threshold contributions in $e^{2} \Pi_{\gamma \gamma}^{(t)}(0)$ and $\operatorname{Re} A_{\gamma Z}\left(m_{Z}^{2}\right) / m_{Z}^{2}$ are very small, we have not subtracted them from $\Delta_{\gamma}$ or the latter amplitude. Consequently, they do not affect the counterterms of Eqs. (7d) and (9a).

Analogous considerations are valid in the on-shell evaluation of $\Delta r$ (see Sec. V and Ref. [31]). The threshold corrections that are not suppressed by reduced-mass effects occur again in $\Pi_{\gamma \gamma}^{(t)}(0)$ and $\operatorname{Re} A_{Z Z}^{(t)}\left(m_{Z}^{2}\right) / m_{Z}^{2}$, the latter being by far the dominant contribution for large $m_{t}$. Here one sets $\hat{s}^{2} \rightarrow s^{2}$ and $\hat{c}^{2} \rightarrow c^{2}$ everywhere. We also recall that in $\Delta r$ the contribution of Eq. (32a) is enhanced by a factor $c^{2} / s^{2}$.

We end this section with the observation that the magnitude of the $1 S$ contribution to $\operatorname{Im}^{V}\left(s, m_{t}, m_{t}\right) / s$ can be roughly understood on the basis of a simplified "Bohratom" model. We recall that, neglecting hard-gluon corrections, this contribution is approximately given by $[24$, 31]

$$
\frac{\operatorname{Im} \Pi^{V}\left(s, m_{t}, m_{t}\right)}{s} \approx 3 \frac{\left|R_{1,0}(0)\right|^{2}}{M_{\theta}} \delta\left(s-M_{\theta}^{2}\right),
$$


where $R_{1,0}(0)$ is the radial wave function at the origin and $M_{\theta} \approx 2 m_{t}$ is the mass of the $1 S$ resonance, $\theta$. In Ref. [24], $\left|R_{1,0}(0)\right|^{2}$ and $M_{\theta}$ have been studied in detail on the basis of the Richardson potential. Suppose now that we attempt to estimate this effect using the "Bohr-atom" picture. In the case of the one-electron atom, $\left[R_{1,0}(0)\right]^{2}=4(\alpha \mu)^{3}$, where $\mu$ is the reduced mass. For toponium we set $\mu=m_{t} / 2, M_{\theta}=2 m_{t}$, and replace $\alpha \rightarrow 4 \hat{\alpha}_{s}\left(k_{1}\right) / 3$, where $k_{1}=2 \hat{\alpha}_{s}\left(k_{1}\right) m_{t} / 3$ is the inverse Bohr radius [27]. This leads to $3\left|R_{1,0}(0)\right|^{2} /\left(M_{\theta} m_{Z}^{2}\right)=$ $(16 / 9)\left(\hat{\alpha}_{s}\left(k_{1}\right)\right)^{3} m_{t}^{2} / m_{Z}^{2}$, which is to be compared with $3 m_{t} \xi\left(m_{t}\right) /\left(8 m_{Z}^{2}\right)$, obtained from the Richardson potential $[24,31]$. Here $\xi\left(m_{t}\right)$ is a monotonically increasing function of $m_{t}$, which is evaluated numerically and varies from 1.95 to $3.08 \mathrm{GeV}$ for $m_{Z} \leq m_{t} \leq 250 \mathrm{GeV}$. For $m_{t}=m_{Z}, 250 \mathrm{GeV}$, the Richardson potential gives 0.0080 and 0.0347 , respectively, while in the Bohr-atom model the corresponding values are 0.0096 and 0.0428 , which are about $20 \%$ larger. This may be partly due to the fact that the Richardson potential is softer than Coulombic near the origin. Interestingly, the ratio of the values at $m_{t}=250 \mathrm{GeV}$ and $m_{t}=m_{Z}$, which gives an indication of the $m_{t}$ dependence, is almost the same in both cases. In the Bohr-atom model, for the $n S$ states, $\left|R_{n, 0}(0)\right|^{2}$ scales as $n^{-3}$. However, as the radii of the higher orbits increase as $n^{2}$, the momentum $k_{n}$ at which $\hat{\alpha}_{s}$ is to be evaluated becomes smaller. For sufficiently high $n$, the relevant $\hat{\alpha}_{s}$ falls in the nonperturbative regime and the "Coulombic" picture becomes increasingly doubtful. The evaluation based on a realistic, "confining" potential is clearly preferable. However, it is interesting that a simple Coulomb potential gives a similar answer, at least in the case of the $1 S$ state.

\section{NUMERICAL CALCULATIONS}

In the previous sections we have discussed the theoretical background necessary for the incorporation of the leading QCD effects in the basic corrections $\Delta \hat{r}_{W}, \Delta \hat{r}$, and $\Delta r$. At the same time, as explained in Sec. I, we have introduced a number of refinements in the analysis of the electroweak corrections.

In this section we apply the previous results to numerically evaluate $\Delta \hat{r}_{W}, \Delta \hat{r}$, and $\Delta r$, and, most importantly, to derive precise values for $m_{W}$ and $\hat{s}^{2} \equiv \sin ^{2} \hat{\theta}_{W}\left(m_{Z}\right)$, as functions of $m_{t}$ and $m_{H}$. Working first in the $\overline{\mathrm{MS}}$ scheme, the basic strategy is the same as in Ref. [1]. We employ Eq. (10c) and Eqs. (14) and (18a) as the basic expressions for $\Delta \hat{r}_{W}$ and $\Delta \hat{r}$, respectively, and iteratively evaluate these corrections in conjunction with Eq. (19a), leading to accurate values for $\hat{s}^{2}$. Then $\Delta r$ can be evaluated from Eqs. (19c) or (19e), $s^{2}$ from Eq. (19b), and $m_{W}$ from either $s^{2}$ or Eq. (19f). We use as input values $\alpha=(137.0359895)^{-1}, G_{\mu}=1.16639 \times 10^{-5} \mathrm{GeV}^{-2}$ [38], $m_{Z}=91.187 \mathrm{GeV}[16]$, leading to $A=\left(\pi \alpha / \sqrt{2} G_{\mu}\right)^{1 / 2}=$ $37.2802 \mathrm{GeV}$, and $\hat{\alpha}_{s}\left(m_{Z}\right)=0.118$ [39]. As shown in Appendix $\mathrm{C}$, the effects arising from finite fermion masses are very minor. Nonetheless, we include them as follows: the $u, d$, and $s$ quarks are treated as massless, while we employ $m_{c}=1.5 \mathrm{GeV}$ and $m_{b}=4.5 \mathrm{GeV}$. The lep- tons are given their physical masses [38], including the recent value $m_{\tau}=1.777 \mathrm{GeV}$ [51]. In order to incorporate the new results on the leading irreducible corrections of $O\left(\alpha^{2}\right)$ [7], it is very convenient to use a precise analytical representation of the function $R\left(m_{H} / m_{t}\right)$ in Eqs. (17a), (18a), and (23b). In the range $r=m_{H} / m_{t}>4$, the authors of Ref. [7] give the accurate asymptotic expansion

$$
\begin{aligned}
R(r)= & \frac{49}{4}+\pi^{2}-27 \ln r+6 \ln ^{2} r \\
& +\frac{1}{3 r^{2}}\left(2-12 \pi^{2}-24 \ln r-108 \ln ^{2} r\right) \\
& +\frac{1}{48 r^{4}}\left(1613-240 \pi^{2}+3000 \ln r-2880 \ln ^{2} r\right)
\end{aligned}
$$

In the complementary domain $0<r \leq 4$, only numerical values are available [7], which we have fitted with the expression

$$
\begin{aligned}
R(r)= & -0.7392088+r(-11.5315+0.382497 \ln r) \\
& +r^{2}\left(5.31338-3.055 \ln r+0.523039 \ln ^{2} r\right)
\end{aligned}
$$

The maximum deviation of Eq. (34b) from the original data [7] is $\lesssim 0.025$ and occurs near the matching point, $r=4$.

In Tables I-V we display the calculated values of $m_{W}$ and $\hat{s}^{2}$, as functions of $m_{t}$ for $m_{H}=(60,100$, $250,600,1000) \mathrm{GeV}$. Currently, a fit to all data, using the electroweak radiative corrections of the SM and $\alpha_{s}\left(m_{Z}\right)=0.120 \pm 0.006$, gives $m_{t}=145_{-19-19}^{+17+17} \mathrm{GeV}$ [51]. The central value corresponds to $m_{H}=300 \mathrm{GeV}$ and the last error reflects the theoretical uncertainty associated with the range $60 \mathrm{GeV} \leq m_{H} \leq 1 \mathrm{TeV}$. This implies $m_{t} \leq 173_{-19}^{+17} \mathrm{GeV}$ at the $95 \%$ confidence level, where the error is again due to the $m_{H}$ uncertainty. Although this strongly suggests that $m_{t} \lesssim 200 \mathrm{GeV}$, in the tables we present values up to $m_{t}=250 \mathrm{GeV}$. One of the reasons is that it is theoretically interesting to study and compare the high- $m_{t}$ dependence of the various radiative corrections. Moreover, there is always the possibility of a statistical surprise or that unknown new physics may alter the predictions of the SM, so that scenarios in which $m_{t}$ is found at higher values are not completely excluded. For comparison purposes, we list in the tables the results of four different calculations: ( $i$ ) only electroweak corrections, with all QCD corrections turned off (columns labeled EW for "electroweak"); (ii) electroweak plus perturbative $O\left(\alpha \alpha_{s}\right)$ corrections (columns labeled $\mathrm{P}$ for "perturbative"); (iii) the above, plus threshold effects calculated in the resonance approach according to Sec. VI (columns labeled $\mathrm{P}+\mathrm{R}$ for "perturbative plus resonance"); ( $i v)$ the same as in (iii) but with threshold effects evaluated in the Green-function approach (columns labeled $\mathrm{P}+\mathrm{G}$ for "perturbative plus Green function"). In order to keep the tables compact, we have displayed only the quantities of greatest physical interest, namely $m_{W}$ and $\hat{s}^{2}$, rather than the radiative corrections $\Delta \hat{r}_{W}$, $\Delta \hat{r}$, and $\Delta r$, or the derived parameter $s^{2}$ of the on-shell scheme. The interested reader can readily glean these important quantities from the tables. Thus, inserting 
TABLE I. Calculated values of $\sin ^{2} \hat{\theta}_{W}\left(m_{Z}\right)$ and $m_{W}$, as a function of $m_{t}$, for $m_{Z}=91.187 \mathrm{GeV}, \hat{\alpha}_{s}\left(m_{Z}\right)=0.118$, and $m_{H}=60 \mathrm{GeV}$. The $\mathrm{EW}$ column includes only electroweak radiative corrections. The $\mathrm{P}$ column incorporates perturbative $O\left(\alpha \alpha_{s}\right)$ contributions (see Sec. III). The P+R and $\mathrm{P}+\mathrm{G}$ columns contain also $t \bar{t}$ threshold effects (see Sec. VI) in the resonance and Green-function approaches, respectively. The on-shell parameter $\sin ^{2} \theta_{W}$ and the radiative corrections $\Delta \hat{r}_{W}, \Delta \hat{r}$, and $\Delta r$ can be gleaned from this table, as explained in Sec. VII.

\begin{tabular}{|c|c|c|c|c|c|c|c|c|}
\hline \multirow{3}{*}{$\begin{array}{c}m_{t} \\
(\mathrm{GeV})\end{array}$} & \multicolumn{4}{|c|}{$\hat{s}^{2}$} & \multicolumn{4}{|c|}{$m_{W}(\mathrm{GeV})$} \\
\hline & \multirow{2}{*}{ EW } & \multicolumn{3}{|c|}{$\mathrm{EW}+\mathrm{QCD}$} & \multirow{2}{*}{ EW } & \multicolumn{3}{|c|}{$\mathrm{EW}+\mathrm{QCD}$} \\
\hline & & $\mathrm{P}$ & $\mathrm{P}+\mathrm{R}$ & $\mathrm{P}+\mathrm{G}$ & & $\bar{P}$ & $\mathrm{P}+\mathrm{R}$ & $\overline{\mathrm{P}+\mathrm{G}}$ \\
\hline$m_{Z}$ & 0.23307 & 0.23321 & 0.23327 & 0.23326 & 79.979 & 79.947 & 79.938 & 79.941 \\
\hline 100 & 0.23287 & 0.23303 & 0.23309 & 0.23307 & 80.029 & 79.996 & 79.986 & 79.989 \\
\hline 110 & 0.23262 & 0.23280 & 0.23287 & 0.23285 & 80.085 & 80.050 & 80.038 & 80.041 \\
\hline 120 & 0.23235 & 0.23255 & 0.23263 & 0.23261 & 80.141 & 80.103 & 80.091 & 80.094 \\
\hline 130 & 0.23207 & 0.23229 & 0.23238 & 0.23236 & 80.200 & 80.158 & 80.144 & 80.148 \\
\hline 140 & 0.23176 & 0.23201 & 0.23211 & 0.23209 & 80.260 & 80.215 & 80.199 & 80.203 \\
\hline 150 & 0.23144 & 0.23172 & 0.23182 & 0.23180 & 80.323 & 80.274 & 80.256 & 80.260 \\
\hline 160 & 0.23110 & 0.23140 & 0.23152 & 0.23150 & 80.389 & 80.335 & 80.316 & 80.319 \\
\hline 170 & 0.23074 & 0.23108 & 0.23120 & 0.23118 & 80.457 & 80.398 & 80.377 & 80.380 \\
\hline 180 & 0.23036 & 0.23073 & 0.23087 & 0.23085 & 80.528 & 80.463 & 80.441 & 80.444 \\
\hline 190 & 0.22997 & 0.23037 & 0.23052 & 0.23050 & 80.603 & 80.532 & 80.507 & 80.510 \\
\hline 200 & 0.22955 & 0.22999 & 0.23015 & 0.23013 & 80.680 & 80.603 & 80.576 & 80.578 \\
\hline 210 & 0.22912 & 0.22959 & 0.22976 & 0.22976 & 80.760 & 80.677 & 80.648 & 80.650 \\
\hline 220 & 0.22867 & 0.22918 & 0.22936 & 0.22936 & 80.844 & 80.754 & 80.723 & 80.723 \\
\hline 230 & 0.22820 & 0.22875 & 0.22895 & 0.22895 & 80.930 & 80.833 & 80.800 & 80.799 \\
\hline 240 & 0.22772 & 0.22831 & 0.22851 & 0.22853 & 81.020 & 80.916 & 80.881 & 80.878 \\
\hline 250 & 0.22721 & 0.22785 & 0.22806 & 0.22809 & 81.114 & 81.001 & 80.964 & 80.959 \\
\hline
\end{tabular}

the value of $\hat{s}^{2}$ in Eq. (3) and those of $\hat{s}^{2}$ and $m_{W}$ in Eq. (2), one finds $\Delta \hat{r}$ and $\Delta \hat{r}_{W}$, respectively. Using $m_{W}$, one calculates $s^{2} \equiv 1-m_{W}^{2} / m_{Z}^{2}$ and, in conjunction with Eq. (1), $\Delta r$. We have kept enough decimal figures in $\hat{s}^{2}$ and $m_{W}$, so that $\Delta \hat{r}_{W}, \Delta \hat{r}, \Delta r$, and $s^{2}$ can be accurately evaluated. The tables allow us to separate the threshold effects from the more established perturbative $O\left(\alpha \alpha_{s}\right)$ contributions. Although the resonance (R) and Greenfunction (G) approaches are quite different conceptually and technically, the tables reveal the welcome and rather surprising result that their effect on $m_{W}$ and $\hat{s}^{2}$ is very similar over the entire range $m_{Z} \leq m_{t} \leq 250 \mathrm{GeV}$, $60 \mathrm{GeV} \leq m_{H} \leq 1 \mathrm{TeV}$. Nonetheless, for reasons explained in Ref. [31] and Sec. VI, in our specific applications we use the resonance method for $m_{t} \lesssim 130 \mathrm{GeV}$ and the Green-function approach for $m_{t} \gtrsim 130 \mathrm{GeV}$.

It is also a curious and rather surprising fact that most of the new effects we have considered relative to Ref.

TABLE II. As Table I, for $m_{H}=100 \mathrm{GeV}$.

\begin{tabular}{|c|c|c|c|c|c|c|c|c|}
\hline \multirow{3}{*}{$\begin{array}{c}m_{t} \\
(\mathrm{GeV})\end{array}$} & \multicolumn{4}{|c|}{$\hat{s}^{2}$} & \multicolumn{4}{|c|}{$m_{W}(\mathrm{GeV})$} \\
\hline & \multirow{2}{*}{ EW } & \multicolumn{3}{|c|}{$\mathrm{EW}+\mathrm{QCD}$} & \multirow{2}{*}{ EW } & \multicolumn{3}{|c|}{$\mathrm{EW}+\mathrm{QCD}$} \\
\hline & & $\mathrm{P}$ & $\mathrm{P}+\mathrm{R}$ & $\mathrm{P}+\mathrm{G}$ & & $\mathrm{P}$ & $\mathrm{P}+\mathrm{R}$ & $\overline{\mathrm{P}+\mathrm{G}}$ \\
\hline$m_{Z}$ & 0.23332 & 0.23346 & 0.23352 & 0.23351 & 79.952 & 79.921 & 79.912 & 79.914 \\
\hline 100 & 0.23312 & 0.23328 & 0.23334 & 0.23332 & 80.002 & 79.969 & 79.960 & 79.962 \\
\hline 110 & 0.23287 & 0.23305 & 0.23312 & 0.23310 & 80.058 & 80.023 & 80.012 & 80.015 \\
\hline 120 & 0.23260 & 0.23280 & 0.23289 & 0.23287 & 80.115 & 80.077 & 80.064 & 80.068 \\
\hline 130 & 0.23232 & 0.23254 & 0.23263 & 0.23261 & 80.173 & 80.132 & 80.118 & 80.121 \\
\hline 140 & 0.23201 & 0.23227 & 0.23236 & 0.23234 & 80.234 & 80.188 & 80.172 & 80.176 \\
\hline 150 & 0.23169 & 0.23197 & 0.23208 & 0.23206 & 80.296 & 80.246 & 80.229 & 80.233 \\
\hline 160 & 0.23135 & 0.23166 & 0.23178 & 0.23175 & 80.361 & 80.307 & 80.288 & 80.292 \\
\hline 170 & 0.23100 & 0.23133 & 0.23146 & 0.23144 & 80.429 & 80.370 & 80.349 & 80.352 \\
\hline 180 & 0.23062 & 0.23099 & 0.23113 & 0.23111 & 80.500 & 80.435 & 80.412 & 80.416 \\
\hline 190 & 0.23023 & 0.23063 & 0.23078 & 0.23076 & 80.574 & 80.503 & 80.478 & 80.481 \\
\hline 200 & 0.22982 & 0.23025 & 0.23041 & 0.23040 & 80.651 & 80.574 & 80.547 & 80.549 \\
\hline 210 & 0.22939 & 0.22986 & 0.23003 & 0.23002 & 80.730 & 80.647 & 80.618 & 80.619 \\
\hline 220 & 0.22894 & 0.22945 & 0.22964 & 0.22963 & 80.813 & 80.723 & 80.692 & 80.692 \\
\hline 230 & 0.22848 & 0.22903 & 0.22922 & 0.22923 & 80.899 & 80.801 & 80.769 & 80.767 \\
\hline 240 & 0.22800 & 0.22859 & 0.22879 & 0.22881 & 80.988 & 80.883 & 80.848 & 80.845 \\
\hline 250 & 0.22750 & 0.22814 & 0.22835 & 0.22838 & 81.080 & 80.967 & 80.930 & 80.925 \\
\hline
\end{tabular}


TABLE III. As Table I, for $m_{H}=250 \mathrm{GeV}$.

\begin{tabular}{|c|c|c|c|c|c|c|c|c|}
\hline \multirow{3}{*}{$\begin{array}{c}m_{t} \\
(\mathrm{GeV})\end{array}$} & \multicolumn{4}{|c|}{$\hat{s}^{2}$} & \multicolumn{4}{|c|}{$m_{W}(\mathrm{GeV})$} \\
\hline & \multirow{2}{*}{ EW } & \multicolumn{3}{|c|}{$\mathrm{EW}+\mathrm{QCD}$} & \multirow{2}{*}{ EW } & \multicolumn{3}{|c|}{$\mathrm{EW}+\mathrm{QCD}$} \\
\hline & & $\mathrm{P}$ & $\mathrm{P}+\mathrm{R}$ & $\mathrm{P}+\mathrm{G}$ & & $\mathrm{P}$ & $\mathrm{P}+\mathrm{R}$ & $\overline{P+G}$ \\
\hline$m_{Z}$ & 0.23380 & 0.23394 & 0.23401 & 0.23399 & 79.894 & 79.862 & 79.854 & 79.856 \\
\hline 100 & 0.23360 & 0.23376 & 0.23383 & 0.23381 & 79.944 & 79.911 & 79.901 & 79.904 \\
\hline 110 & 0.23335 & 0.23353 & 0.23361 & 0.23359 & 80.000 & 79.965 & 79.953 & 79.956 \\
\hline 120 & 0.23308 & 0.23329 & 0.23337 & 0.23335 & 80.056 & 80.018 & 80.005 & 80.009 \\
\hline 130 & 0.23280 & 0.23303 & 0.23312 & 0.23310 & 80.114 & 80.072 & 80.058 & 80.062 \\
\hline 140 & 0.23250 & 0.23275 & 0.23285 & 0.23283 & 80.174 & 80.128 & 80.113 & 80.116 \\
\hline 150 & 0.23218 & 0.23246 & 0.23257 & 0.23255 & 80.236 & 80.186 & 80.169 & 80.172 \\
\hline 160 & 0.23185 & 0.23215 & 0.23227 & 0.23225 & 80.301 & 80.246 & 80.227 & 80.230 \\
\hline 170 & 0.23149 & 0.23183 & 0.23196 & 0.23194 & 80.368 & 80.308 & 80.287 & 80.290 \\
\hline 180 & 0.23112 & 0.23149 & 0.23163 & 0.23161 & 80.437 & 80.372 & 80.349 & 80.352 \\
\hline 190 & 0.23073 & 0.23114 & 0.23129 & 0.23127 & 80.510 & 80.439 & 80.414 & 80.417 \\
\hline 200 & 0.23033 & 0.23077 & 0.23093 & 0.23092 & 80.585 & 80.508 & 80.481 & 80.483 \\
\hline 210 & 0.22991 & 0.23039 & 0.23056 & 0.23055 & 80.663 & 80.579 & 80.550 & 80.552 \\
\hline 220 & 0.22947 & 0.22999 & 0.23017 & 0.23017 & 80.744 & 80.653 & 80.622 & 80.622 \\
\hline 230 & 0.22902 & 0.22958 & 0.22977 & 0.22978 & 80.827 & 80.730 & 80.697 & 80.695 \\
\hline 240 & 0.22855 & 0.22915 & 0.22936 & 0.22937 & 80.914 & 80.809 & 80.773 & 80.771 \\
\hline 250 & 0.22807 & 0.22871 & 0.22893 & 0.22896 & 81.003 & 80.890 & 80.853 & 80.848 \\
\hline
\end{tabular}

[1], namely (a) the incorporation of the recent results on the leading irreducible corrections of $O\left(\alpha^{2}\right),(b)$ the perturbative $O\left(\alpha \alpha_{s}\right)$ corrections, and $(c)$ the threshold contributions in the formulation of Sec. VI, increase the values of $\Delta \hat{r}$ and $\Delta r$ for given $m_{t}$ and $m_{H}$. Thus, they have a sign opposite to that of the leading $m_{t}$-dependent part of the one-loop corrections and, therefore, they induce an increase in the $m_{t}$ upper bound. For large $m_{t}$, they are only partially compensated by the shift of $-5 \times 10^{-4}$ in $\Delta \hat{r}$ and $\Delta r$ arising from the new calculation of $e^{2} \operatorname{Re}\left[\Pi_{\gamma \gamma}^{(5)}(0)-\Pi_{\gamma \gamma}^{(5)}\left(m_{Z}^{2}\right)\right]$ [10]. It should also be remembered that most of the corresponding shifts in $\Delta \hat{r}$ and $\Delta r$ increase with $m_{t}:(a)$ behaves as $m_{t}^{4}$ [cf.
Eqs. (17a), (18a), and (23b)], the dominant (b) contributions as $m_{t}^{2}$ [cf. Eqs. (16a) and (19c)], and (c) more rapidly than linearly in $m_{t}$ [31].

The QCD effects on $m_{W}$ and $\hat{s}^{2}$ are visible in the tables. For example, for the intermediate value $m_{H}=$ $250 \mathrm{GeV}$ and $m_{t}=m_{Z}, 130,150,180,200,250 \mathrm{GeV}$, the perturbative $O\left(\alpha \alpha_{s}\right)$ corrections lead to the shifts

$$
\left.\begin{array}{rl}
\Delta m_{W} & =-(32,42,50,65,77,113) \mathrm{MeV} \\
\Delta \hat{s}^{2} & =+(1.4,2.3,2.8,3.7,4.4,6.4) \times 10^{-4}
\end{array}\right\} O\left(\alpha \alpha_{s}\right) .
$$

The threshold contributions lead to further shifts

TABLE IV. As Table I, for $m_{H}=600 \mathrm{GeV}$.

\begin{tabular}{|c|c|c|c|c|c|c|c|c|}
\hline \multirow{3}{*}{$\begin{array}{c}m_{t} \\
(\mathrm{GeV})\end{array}$} & \multicolumn{4}{|c|}{$\hat{s}^{2}$} & \multicolumn{4}{|c|}{$m_{W}(\mathrm{GeV})$} \\
\hline & \multirow{2}{*}{ EW } & \multicolumn{3}{|c|}{$\mathrm{EW}+\mathrm{QCD}$} & \multirow{2}{*}{ EW } & \multicolumn{3}{|c|}{ EW + QCD } \\
\hline & & $\mathrm{P}$ & $\mathrm{P}+\mathrm{R}$ & $P+G$ & & $\mathbf{P}$ & $\mathrm{P}+\mathrm{R}$ & $\overline{P+G}$ \\
\hline$\overline{m_{Z}}$ & 0.23430 & 0.23444 & 0.23450 & 0.23448 & 79.828 & 79.796 & 79.787 & 79.790 \\
\hline 100 & 0.23409 & 0.23425 & 0.23432 & 0.23430 & 79.878 & 79.844 & 79.835 & 79.837 \\
\hline 110 & 0.23384 & 0.23403 & 0.23410 & 0.23408 & 79.933 & 79.898 & 79.887 & 79.890 \\
\hline 120 & 0.23358 & 0.23378 & 0.23386 & 0.23384 & 79.990 & 79.951 & 79.939 & 79.942 \\
\hline 130 & 0.23329 & 0.23352 & 0.23361 & 0.23359 & 80.047 & 80.005 & 79.991 & 79.995 \\
\hline 140 & 0.23299 & 0.23325 & 0.23335 & 0.23333 & 80.107 & 80.061 & 80.045 & 80.049 \\
\hline 150 & 0.23268 & 0.23296 & 0.23307 & 0.23305 & 80.168 & 80.118 & 80.101 & 80.104 \\
\hline 160 & 0.23234 & 0.23265 & 0.23277 & 0.23275 & 80.232 & 80.177 & 80.158 & 80.162 \\
\hline 170 & 0.23200 & 0.23234 & 0.23247 & 0.23244 & 80.298 & 80.238 & 80.217 & 80.221 \\
\hline 180 & 0.23163 & 0.23200 & 0.23214 & 0.23212 & 80.367 & 80.301 & 80.278 & 80.282 \\
\hline 190 & 0.23125 & 0.23166 & 0.23181 & 0.23179 & 80.438 & 80.367 & 80.342 & 80.344 \\
\hline 200 & 0.23086 & 0.23130 & 0.23146 & 0.23145 & 80.511 & 80.434 & 80.407 & 80.409 \\
\hline 210 & 0.23045 & 0.23093 & 0.23110 & 0.23109 & 80.587 & 80.503 & 80.475 & 80.476 \\
\hline 220 & 0.23002 & 0.23054 & 0.23072 & 0.23072 & 80.666 & 80.575 & 80.544 & 80.544 \\
\hline 230 & 0.22958 & 0.23014 & 0.23034 & 0.23034 & 80.747 & 80.649 & 80.616 & 80.615 \\
\hline 240 & 0.22913 & 0.22973 & 0.22994 & 0.22995 & 80.830 & 80.725 & 80.689 & 80.687 \\
\hline 250 & 0.22867 & 0.22931 & 0.22953 & 0.22956 & 80.916 & 80.803 & 80.765 & 80.760 \\
\hline
\end{tabular}


TABLE V. As Table I, for $m_{H}=1000 \mathrm{GeV}$.

\begin{tabular}{|c|c|c|c|c|c|c|c|c|}
\hline \multirow{3}{*}{$\begin{array}{c}m_{t} \\
(\mathrm{GeV})\end{array}$} & \multicolumn{4}{|c|}{$\hat{s}^{2}$} & \multicolumn{4}{|c|}{$m_{W}(\mathrm{GeV})$} \\
\hline & \multirow{2}{*}{ EW } & \multicolumn{3}{|c|}{$\mathrm{EW}+\mathrm{QCD}$} & \multirow{2}{*}{ EW } & \multicolumn{3}{|c|}{$\mathrm{EW}+\mathrm{QCD}$} \\
\hline & & $\mathrm{P}$ & $\mathrm{P}+\mathrm{R}$ & $\mathrm{P}+\mathrm{G}$ & & $\mathrm{P}$ & $\mathrm{P}+\mathrm{R}$ & $\overline{\mathrm{P}+\mathrm{G}}$ \\
\hline$m_{Z}$ & 0.23460 & 0.23474 & 0.23480 & 0.23479 & 79.786 & 79.754 & 79.745 & $\overline{79.748}$ \\
\hline 100 & 0.23439 & 0.23455 & 0.23462 & 0.23460 & 79.836 & 79.803 & 79.793 & 79.796 \\
\hline 110 & 0.23414 & 0.23432 & 0.23440 & 0.23438 & 79.892 & 79.856 & 79.845 & 79.848 \\
\hline 120 & 0.23388 & 0.23408 & 0.23416 & 0.23414 & 79.948 & 79.910 & 79.897 & 79.900 \\
\hline 130 & 0.23359 & 0.23382 & 0.23391 & 0.23389 & 80.006 & 79.964 & 79.950 & 79.953 \\
\hline 140 & 0.23329 & 0.23355 & 0.23365 & 0.23362 & 80.065 & 80.019 & 80.004 & 80.007 \\
\hline 150 & 0.23297 & 0.23326 & 0.23337 & 0.23334 & 80.127 & 80.077 & 80.059 & 80.063 \\
\hline 160 & 0.23264 & 0.23295 & 0.23307 & 0.23305 & 80.190 & 80.136 & 80.116 & 80.120 \\
\hline 170 & 0.23229 & 0.23264 & 0.23276 & 0.23274 & 80.256 & 80.196 & 80.175 & 80.179 \\
\hline 180 & 0.23193 & 0.23230 & 0.23244 & 0.23242 & 80.325 & 80.259 & 80.236 & 80.239 \\
\hline 190 & 0.23155 & 0.23196 & 0.23211 & 0.23209 & 80.395 & 80.324 & 80.299 & 80.302 \\
\hline 200 & 0.23116 & 0.23160 & 0.23176 & 0.23175 & 80.468 & 80.390 & 80.363 & 80.366 \\
\hline 210 & 0.23075 & 0.23123 & 0.23141 & 0.23140 & 80.543 & 80.459 & 80.430 & 80.431 \\
\hline 220 & 0.23033 & 0.23085 & 0.23104 & 0.23104 & 80.621 & 80.530 & 80.499 & 80.499 \\
\hline 230 & 0.22990 & 0.23046 & 0.23066 & 0.23066 & 80.700 & 80.602 & 80.569 & 80.568 \\
\hline 240 & 0.22946 & 0.23006 & 0.23027 & 0.23028 & 80.782 & 80.677 & 80.641 & 80.638 \\
\hline 250 & 0.22900 & 0.22965 & 0.22987 & 0.22990 & 80.866 & 80.753 & 80.715 & 80.710 \\
\hline
\end{tabular}

$\left.\begin{array}{rl}\Delta m_{W} & =-(8,14,14,20,25,42) \mathrm{MeV} \\ \Delta \hat{s}^{2} & =+(0.7,0.9,0.9,1.2,1.5,2.5) \times 10^{-4}\end{array}\right\}$ (threshold $)$

The departure from the monotonic behavior between $m_{t}=130 \mathrm{GeV}$ and $m_{t}=150 \mathrm{GeV}$ is due to the transition from the resonance to the Green-function approach. The effect of the leading irreducible corrections of $O\left(\left(\alpha m_{t}^{2} / m_{W}^{2}\right)^{2}\right)$ cannot be seen in the tables because they have been included in every column. However, they can be estimated from the relations

$$
\begin{aligned}
\frac{\Delta m_{W}}{m_{W}} & \approx \frac{c^{2}}{c^{2}-s^{2}-2 c^{2} x_{t}} R\left(\frac{m_{H}}{m_{t}}\right) \frac{x_{t}^{2}}{6}, \\
\Delta \hat{s}^{2} & \approx-\frac{\hat{s}^{2} \hat{c}^{2}}{\hat{c}^{2}-\hat{s}^{2}} R\left(\frac{m_{H}}{m_{t}}\right) \frac{x_{t}^{2}}{3\left(1-x_{t}\right)^{2}},
\end{aligned}
$$

where $x_{t}$ is defined in Eq. (17b). The term $2 c^{2} x_{t}$ in the first denominator of Eq. (36a) and the factor $\left(1-x_{t}\right)^{-2}$ in Eq. (36b) take into account the fact that some of the leading one-loop contributions to $\Delta r$ and $\Delta \hat{r}$ depend on $s^{2}$ and $\hat{s}^{2}$, respectively, and are therefore affected by the shifts in these parameters. For the same values of $m_{H}$ and $m_{t}$ as employed before, Eqs. (36a) and (36b) give

$$
\left.\begin{array}{c}
\Delta m_{W}=c \\
\Delta \hat{s}^{2}=+(0.1,0.3,0.5,0.9,1.4,3.0) \times 10^{-4}
\end{array}\right\} O\left(\alpha^{2}\right)
$$

Equations (35a), (35b), and (36c) can be compared with the experimental uncertainties $\left(\delta m_{W}\right)_{\text {expt }} \approx 100 \mathrm{MeV}$ and $\left(\delta \hat{s}^{2}\right)_{\text {expt }} \approx 4 \times 10^{-4}$ expected at the end of 1993 [51]. We see that, in general terms, they are of the same order of magnitude. Of course, in the long run, even better experimental accuracies are envisaged, reaching per- haps $\left(\delta m_{W}\right)_{\text {expt }} \approx 50 \mathrm{MeV}$. The above shifts can also be compared with the theoretical uncertainties

$$
\begin{aligned}
\left(\delta m_{W}\right)_{\mathrm{th}} & =-\frac{m_{W}}{2} \frac{s^{2}}{c^{2}-s^{2}-2 c^{2} x_{t}} \frac{\delta \Delta r}{1-\Delta \alpha} \\
& \approx \pm 16 \mathrm{MeV}, \\
\left(\delta \hat{s}^{2}\right)_{\mathrm{th}} & =\frac{\hat{c}^{2} \hat{s}^{2}}{\left(\hat{c}^{2}-\hat{s}^{2}\right)} \frac{\delta \Delta \hat{r}}{1-\Delta_{\gamma}} \approx \pm 3 \times 10^{-4},
\end{aligned}
$$

arising from the error $(\delta \Delta r)_{\mathrm{th}} \approx(\delta \Delta \hat{r})_{\mathrm{th}}= \pm 9 \times 10^{-4}$ in the calculation of $e^{2} \operatorname{Re}\left[\Pi_{\gamma \gamma}^{(5)}(0)-\Pi_{\gamma \gamma}^{(5)}\left(m_{Z}^{2}\right)\right]$ [10]. We recall that the theoretical error arising from the neglect of higher-order electroweak corrections is expected to be of $O\left(\left(\alpha / \pi s^{2}\right) x_{t}\right)$ in $\Delta \hat{r}_{W}$ and $\Delta \hat{r}$ and further enhanced by a factor $c^{2} / s^{2}$ in $\Delta r$ (see Sec. I). Moreover, the error in the overall QCD corrections (perturbative and threshold effects) has been estimated to be $\pm 20 \%$ [31]. Incidentally, Eq. (37b) shows that, if the experimental accuracy in $\hat{s}^{2}$ is improved in the future well beyond $4 \times 10^{-4}$, a meaningful theoretical interpretation will require a decrease in the above-mentioned theoretical errors.

As pointed out before, the higher-order corrections we have considered lead to an increase in the $m_{t}$ values obtained from experiments. As an example, we consider the case $m_{H}=250 \mathrm{GeV}$ and call $m_{t}$ the parameter derived from $\left(m_{W}\right)_{\text {expt }}$ when the perturbative $O\left(\alpha \alpha_{s}\right)$ corrections are included. Table III shows that the possible values $m_{t}=m_{Z}, 130,150,180,200 \mathrm{GeV}$ are larger than those obtained in the purely EW calculation by

$\Delta m_{t}=+(5.8,7.5,8.3,9.7,10.8) \mathrm{GeV} \quad\left[O\left(\alpha \alpha_{s}\right)\right]$.

The values of $m_{t}$ derived from $\left(\hat{s}^{2}\right)$ expt are shifted by slightly higher amounts, the differences with Eq.(38a) being $\lesssim 0.8 \mathrm{GeV}$. The bulk of $\Delta m_{t}$ arising from the perturbative $O\left(\alpha \alpha_{s}\right)$ corrections can be understood with the simple formula (16c), which describes the dominant 
TABLE VI. Comparison between the values of $\Delta r$ and $m_{W}$ obtained using the $\overline{\mathrm{MS}}$ approach of Sec. III $\left[(\Delta r)_{\mathrm{I}}\right.$, $\left.\left(m_{W}\right)_{\mathrm{I}}\right]$ and the on-shell formulation of Sec. V $\left[(\Delta r)_{\mathrm{II}}\right.$, $\left.\left(m_{W}\right)_{\mathrm{II}}\right]$, as a function of $m_{t}$, for $m_{Z}=91.187 \mathrm{GeV}$ and $m_{H}=60 \mathrm{GeV}$. Nonperturbative $t \bar{t}$ threshold effects are not included here.

\begin{tabular}{|c|c|c|c|c|}
\hline $\begin{array}{c}m_{t} \\
(\mathrm{GeV})\end{array}$ & \multicolumn{2}{|c|}{$\times 10^{2}$} & $\left(m_{W}\right)_{\mathrm{I}}$ & $\left(m_{W}\right)_{\mathrm{II}}$ \\
\hline$m_{Z}$ & 6.003 & 6.006 & 79.947 & 79.946 \\
\hline 100 & 5.735 & 5.738 & 79.996 & 79.995 \\
\hline 110 & 5.437 & 5.440 & 80.050 & 80.049 \\
\hline 120 & 5.135 & 5.138 & 80.103 & 80.103 \\
\hline 130 & 4.823 & 4.826 & 80.158 & 80.158 \\
\hline 140 & 4.498 & 4.502 & 80.215 & 80.214 \\
\hline 150 & 4.159 & 4.162 & 80.274 & 80.273 \\
\hline 160 & 3.801 & 3.805 & 80.334 & 80.334 \\
\hline 170 & 3.425 & 3.429 & 80.398 & 80.397 \\
\hline 180 & 3.029 & 3.033 & 80.463 & 80.463 \\
\hline 190 & 2.611 & 2.615 & 80.532 & 80.531 \\
\hline 200 & 2.170 & 2.173 & 80.603 & 80.602 \\
\hline 210 & 1.705 & 1.708 & 80.677 & 80.676 \\
\hline 220 & 1.214 & 1.217 & 80.754 & 80.753 \\
\hline 230 & 0.697 & 0.699 & 80.833 & 80.833 \\
\hline 240 & 0.151 & 0.153 & 80.916 & 80.915 \\
\hline 250 & -0.424 & -0.423 & 81.001 & 81.001 \\
\hline
\end{tabular}

contribution. Similarly, we see from Table III that, when threshold contributions are included, the values of $m_{t}$ derived from $\left(m_{W}\right)$ expt are larger than those obtained in the $\mathrm{EW}+\mathrm{P}$ calculation by additional shifts of

$$
\Delta m_{t}=+(1.7,2.7,2.7,3.3,4.1) \mathrm{GeV} \quad \text { [threshold] } .
$$

The corresponding variations arising from the irreducible $O\left(\alpha^{2}\right)$ corrections are

$$
\Delta m_{t}=+(0.6,1.2,1.6,2.5,3.3) \mathrm{GeV} \quad\left[O\left(\alpha^{2}\right)\right] .
$$

TABLE VII. As Table VI, for $m_{H}=250 \mathrm{GeV}$.

\begin{tabular}{c|cc|cc}
\hline \hline $\begin{array}{c}m_{t} \\
(\mathrm{GeV})\end{array}$ & $(\Delta r)_{\mathrm{I}}$ & $(\Delta r)_{\mathrm{II}}$ & \multicolumn{2}{c}{$\left(m_{W}\right)_{\mathrm{I}}$} \\
$(\mathrm{GeV})$ & $\left(m_{W}\right)_{\mathrm{II}}$ \\
\hline$m_{Z}$ & 6.461 & 6.462 & 79.862 & 79.862 \\
100 & 6.199 & 6.200 & 79.911 & 79.911 \\
110 & 5.907 & 5.908 & 79.965 & 79.964 \\
120 & 5.612 & 5.613 & 80.018 & 80.018 \\
130 & 5.309 & 5.309 & 80.072 & 80.072 \\
140 & 4.994 & 4.994 & 80.128 & 80.128 \\
150 & 4.665 & 4.665 & 80.186 & 80.186 \\
160 & 4.320 & 4.320 & 80.246 & 80.246 \\
170 & 3.959 & 3.957 & 80.308 & 80.308 \\
180 & 3.579 & 3.577 & 80.372 & 80.372 \\
190 & 3.179 & 3.176 & 80.439 & 80.439 \\
200 & 2.760 & 2.756 & 80.508 & 80.508 \\
210 & 2.319 & 2.313 & 80.579 & 80.580 \\
220 & 1.855 & 1.848 & 80.653 & 80.654 \\
230 & 1.369 & 1.360 & 80.730 & 80.731 \\
240 & 0.858 & 0.846 & 80.809 & 80.810 \\
250 & 0.322 & 0.307 & 80.890 & 80.892 \\
\hline \hline
\end{tabular}

TABLE VIII. As Table VI, for $m_{H}=1000 \mathrm{GeV}$.

\begin{tabular}{c|cc|cc}
\hline \hline $\begin{array}{c}m_{t} \\
(\mathrm{GeV})\end{array}$ & $(\Delta r)_{\mathrm{I}}$ & $(\Delta r)_{\mathrm{II}}$ & \multicolumn{2}{c}{$\left(m_{W}\right)_{\mathrm{I}}$} \\
$(\mathrm{GeV})$ & $\left(m_{W}\right)_{\mathrm{II}}$ \\
\hline$m_{Z}$ & 7.036 & 7.037 & 79.754 & 79.754 \\
100 & 6.779 & 6.780 & 79.803 & 79.803 \\
110 & 6.494 & 6.494 & 79.856 & 79.856 \\
120 & 6.206 & 6.205 & 79.910 & 79.910 \\
130 & 5.910 & 5.910 & 79.964 & 79.964 \\
140 & 5.604 & 5.603 & 80.019 & 80.020 \\
150 & 5.286 & 5.284 & 80.077 & 80.077 \\
160 & 4.954 & 4.951 & 80.135 & 80.136 \\
170 & 4.607 & 4.603 & 80.196 & 80.197 \\
180 & 4.244 & 4.239 & 80.259 & 80.260 \\
190 & 3.865 & 3.859 & 80.324 & 80.325 \\
200 & 3.469 & 3.461 & 80.390 & 80.392 \\
210 & 3.056 & 3.045 & 80.459 & 80.461 \\
220 & 2.625 & 2.612 & 80.530 & 80.532 \\
230 & 2.175 & 2.159 & 80.602 & 80.605 \\
240 & 1.707 & 1.687 & 80.677 & 80.680 \\
250 & 1.220 & 1.195 & 80.753 & 80.757 \\
\hline \hline
\end{tabular}

Thus, for $m_{H}=250 \mathrm{GeV}$ and $m_{t}=200 \mathrm{GeV}$, the combination of $O\left(\alpha \alpha_{s}\right)$, threshold, and leading irreducible $O\left(\alpha^{2}\right)$ corrections increases the value of $m_{t}$ derived from $\left(m_{W}\right)_{\text {expt }}$ by $\approx 16.8 \mathrm{GeV}$.

Another topic of considerable interest is the comparison of calculations carried out in the $\overline{\mathrm{MS}}$ and onshell methods of renormalization. This is illustrated for $m_{H}=60,250,1000 \mathrm{GeV}$ in Tables VI-VIII, where we show the evaluation of $\Delta r$, a physical observable, obtained on the basis of the $\overline{M S}$ approach of Sec. III and the on-shell formulation of Sec. V. For the purposes of this study, we have included the electroweak and perturbative $O\left(\alpha \alpha_{s}\right)$ corrections, leaving aside the threshold effects. We also display the derived values of $m_{W}$ and $s^{2}$. Inspection of the tables shows that the two calculations of $\Delta r$ are in excellent agreement over the entire range $60 \mathrm{GeV} \leq m_{H} \leq 1 \mathrm{TeV}, m_{Z} \leq m_{t} \leq 250 \mathrm{GeV}$, with a maximum discrepancy of $\approx 2.5 \times 10^{-4}$ occurring at $m_{t}=250 \mathrm{GeV}$ and $m_{H}=1 \mathrm{TeV}$. Although such accurate agreement may be somewhat fortuitous, it is roughly of the expected order of magnitude, i.e., $O\left(\left(\alpha / \pi \hat{s}^{2}\right)\left(\hat{c}^{2} / \hat{s}^{2}\right) x_{t}\right)$.

\section{ACKNOWLEDGMENTS}

We would like to thank Riccardo Barbieri, Matteo Beccaria, Giuseppe Degrassi, Fred Jegerlehner, Hans Kühn, William Marciano, Alfred Mueller, Lev Okun, Michael Peskin, Thomas Teubner, and Peter Zerwas for valuable communications and discussions. One of us (A.S.) would like to thank the physicists at SISSA, Trieste, Italy, for their kind hospitality during June 1992, when part of his work on this problem was carried out. This research was supported in part by the National Science Foundation under Grant No. PHY-9017585. One of the authors (S.F.) is currently supported by the ICSC World Laboratory and the CERN Theory Division. 


\section{APPENDIX A}

In this appendix we discuss the perturbative corrections of $O\left(\hat{\alpha}_{s}\right)$ to the vacuum polarization functions involving quarks. Defining $\Pi^{V, A}\left(s, m_{1}, m_{2}\right)$ according to Eqs. (26a) and (26b), we expand

$$
\begin{aligned}
\Pi^{V, A}\left(s, m_{1}, m_{2}\right)= & \Pi_{0}^{V, A}\left(s, m_{1}, m_{2}\right) \\
& +\frac{\hat{\alpha}_{s}}{\pi} \Pi_{1}^{V, A}\left(s, m_{1}, m_{2}\right),
\end{aligned}
$$

where $s=q^{2}$ and $m_{1}$ and $m_{2}$ are the masses of the virtual quarks in the loop. The functions $\Pi_{0}^{V, A}\left(s, m_{1}, m_{2}\right)$ have been extensively discussed in the literature. They can be gleaned, for example, from Ref. [41]. In the $O\left(\hat{\alpha}_{s}\right)$ terms we consider two limiting cases: $m_{1}=m_{2}=m$ (as occurs in the $\gamma \gamma, Z Z$, and $Z \gamma$ self-energies) and $m_{1}=m, m_{2}=0$ [as applies, to a very good approximation, to the $(t, b)$ contribution to the $W W$ self-energy].

Comparison of Refs. [22] and [25] leads to the following expressions:

$$
\begin{aligned}
\frac{\pi^{2}}{m^{2}} \Pi_{1}^{V}(s, m, m)= & r\left(-\frac{1}{n-4}-l-4 \zeta(3)+\frac{55}{12}\right)+V_{1}(r) \\
\frac{\pi^{2}}{m^{2}} \Pi_{1}^{A}(s, m, m)= & \frac{6}{(n-4)^{2}}+\frac{2}{n-4}\left(3 l-\frac{r}{2}-\frac{11}{4}\right)+3 l^{2}-\left(r+\frac{11}{2}\right) l \\
& +r\left(-4 \zeta(3)+\frac{55}{12}\right)+6 \zeta(3)+3 \zeta(2)-\frac{11}{8}+A_{1}(r), \\
\frac{\pi^{2}}{m^{2}} \Pi_{1}^{V, A}(s, m, 0)= & \frac{1}{4}\left[\frac{6}{(n-4)^{2}}+\frac{2}{n-4}\left(3 l-\frac{x}{2}-\frac{11}{4}\right)+3 l^{2}-\left(x+\frac{11}{2}\right) l\right. \\
& \left.\quad+x\left(-4 \zeta(3)+\frac{55}{12}\right)+6 \zeta(3)+3 \zeta(2)-\frac{11}{8}\right]+F_{1}(x),
\end{aligned}
$$

where $r \equiv s /\left(4 m^{2}\right), x \equiv s / m^{2}, l \equiv \ln \left(m^{2} / \mu^{\prime}\right)\left(\mu^{\prime}\right.$ is the rescaled 't Hooft mass discussed in Sec. II), $V_{1}(r)$, $A_{1}(r)$, and $F_{1}(x)$ are complicated functions studied in Ref. [25], and the color factor appropriate to $N_{c}=3$ has been explicitly included. We recall that $\zeta(2)=\pi^{2} / 6$ and $\zeta(3)=1.20206 \ldots$. The above expressions can be used to evaluate the $O\left(\hat{\alpha}_{s}\right)$ corrections employed in the text. Thus, the quantity $\Pi_{\gamma \gamma}^{(f)}(0)$ in Sec. II can be written as

$$
\begin{aligned}
\Pi_{\gamma \gamma}^{(f)}(0)= & \Pi_{\gamma \gamma}^{(l)}(0)+\Pi_{\gamma \gamma}^{(t)}(0)+\operatorname{Re} \Pi_{\gamma \gamma}^{(5)}\left(m_{Z}^{2}\right) \\
& +\operatorname{Re}\left[\Pi_{\gamma \gamma}^{(5)}(0)-\Pi_{\gamma \gamma}^{(5)}\left(m_{Z}^{2}\right)\right],
\end{aligned}
$$

where the superscripts $(l),(t)$, and (5) refer to the contributions of the leptons, the top quark, and the first five quark flavors, respectively. It is easy to see that the contribution $\Pi_{\gamma \gamma}^{(q)}(s)$ of quark $q$ to $\Pi_{\gamma \gamma}(s)$ is

$$
\Pi_{\gamma \gamma}^{(q)}(s)=\frac{Q_{q}^{2} \Pi^{V}\left(s, m_{q}, m_{q}\right)}{s},
$$

where $Q_{q}$ is the charge of the quark in units of the positron charge $e$, and $m_{q}$ is its mass. In particular,

$$
\Pi_{\gamma \gamma}^{(t)}(0)=Q_{t}^{2}\left(\Pi^{V}\right)^{\prime}\left(0, m_{t}, m_{t}\right),
$$

where the prime on the rhs denotes differentiation with respect to $s$. Using our Eq. (A2) and Eq. (18) of Ref. [25], we find that the $O\left(\hat{\alpha}_{s}\right)$ part of $\Pi_{\gamma \gamma}^{(t)}(0)$ is

$$
\left(2 \hat{\alpha}_{s} / 9 \pi^{3}\right)\left\{\ln \left(\mu^{\prime} / m_{t}\right)+\frac{15}{8}-[2(n-4)]^{-1}\right\} .
$$

The finite part of this result is included in the second term of Eq. (7a), while the pole contributes to the last. Similarly,

$$
\operatorname{Re} \Pi_{\gamma \gamma}^{(5)}\left(m_{Z}^{2}\right)=\sum_{q \neq t} Q_{q}^{2} \frac{\operatorname{Re} \Pi^{V}\left(m_{Z}^{2}, m_{q}, m_{q}\right)}{m_{Z}^{2}} .
$$

Using Eq. (A2), we see that the $O\left(\hat{\alpha}_{s}\right)$ part of Eq. (A8) is

$$
\left(\hat{\alpha}_{s} / 4 \pi^{3}\right) \sum_{q \neq t} Q_{q}^{2}\left[2 \ln \left(\mu^{\prime} / m_{Z}\right)+f_{2}\left(r_{q}\right)-(n-4)^{-1}\right],
$$

where $r_{q}$ and $f_{2}(r)$ are defined in Sec. II. The finite and pole parts have been included in the third and last terms of Eq. (7a), respectively. As mentioned in Sec. II, for $e^{2} \operatorname{Re}\left[\Pi_{\gamma \gamma}^{(5)}(0)-\Pi_{\gamma \gamma}^{(5)}\left(m_{Z}^{2}\right)\right]$ we employ a recent evaluation [10]. The contributions of $O(\hat{\alpha})$ within the square brackets in the second and third terms of Eq. (7a) are simply obtained from the $O\left(\hat{\alpha}_{s}\right)$ ones by dividing by the quadratic Casimir coefficient $4 / 3$ for the fundamental representation of $\mathrm{SU}(3)_{\mathrm{C}}$, multiplying by an additional factor of $Q_{q}^{2}$, and replacing $\hat{\alpha}_{s} \rightarrow \hat{\alpha}$. The contributions of $O(\hat{\alpha})$ in $\Pi_{\gamma \gamma}^{(l)}(0)$ [first term in Eq. (7a)] can be obtained from those in $\Pi_{\gamma \gamma}^{(t)}(0)$ by changing $m_{t} \rightarrow m_{l}$ and dividing by $3 Q_{t}^{4}$, where the 3 stands for the color factor.

In the approximation of neglecting the squares of mixing angles, the quark contribution to $A_{W W}^{(f)}$ is

$$
\begin{aligned}
& {\left[A_{W W}^{(f)}(s)\right]_{\text {quarks }}=-\frac{1}{8} \sum_{\text {doublets }} } {\left[\Pi^{V}\left(s, m_{1}, m_{2}\right)\right.} \\
&\left.+\Pi^{A}\left(s, m_{1}, m_{2}\right)\right] .
\end{aligned}
$$

In the $O\left(\hat{\alpha}_{s}\right)$ part we approximate $m_{1}=m_{2}=0$ in the $(u, d)$ and $(c, s)$ contributions and $m_{1}=m_{t}, m_{2}=0$ in the $(t, b)$ contribution. In this limit, $\Pi^{A}\left(s, m_{1}, m_{2}\right)=$ 
$\Pi^{V}\left(s, m_{1}, m_{2}\right)$ and we see that the $O\left(\hat{\alpha}_{s}\right)$ part of Eq. (A 8$)$ is

$$
\begin{aligned}
{\left[A_{W W}^{(f)}(s)\right]_{\mathrm{QCD}}=-\frac{1}{4 \pi} } & {\left[2 \hat{\alpha}_{s}\left(m_{Z}\right) \Pi_{1}^{V}(s, 0,0)\right.} \\
& \left.+\hat{\alpha}_{s}\left(m_{t}\right) \Pi_{1}^{V}\left(s, m_{t}, 0\right)\right],
\end{aligned}
$$

where the first and the second terms correspond to the contributions of the light and $(t, b)$ isodoublets, respec- tively. The choice of renormalization scale in $\hat{\alpha}_{s}$ was explained at the end of Sec. II. Employing our Eq. (A4) and Eq. (20) of Ref. [25], we find

$\operatorname{Re} \Pi_{1}^{V, A}(s, 0,0)=-\frac{s}{4 \pi^{2}}\left(\frac{1}{n-4}+\ln \frac{s}{{\mu^{\prime}}^{2}}+4 \zeta(3)-\frac{55}{12}\right)$

$$
\begin{aligned}
\operatorname{Re}\left[\frac{A_{W W}^{(f)}\left(m_{W}^{2}\right)-A_{W W}^{(f)}(0)}{m_{W}^{2}}\right]_{Q C D}= & \frac{\hat{\alpha}_{s}\left(m_{Z}\right)}{8 \pi^{3}}\left(\frac{1}{n-4}+\ln \frac{m_{W}^{2}}{\mu^{\prime 2}}+4 \zeta(3)-\frac{55}{12}\right) \\
& +\frac{\hat{\alpha}_{s}\left(m_{t}\right)}{16 \pi^{3}}\left[\frac{1}{n-4}+\ln \frac{m_{W}^{2}}{\mu^{\prime 2}}+4 \zeta(3)-\frac{55}{12}-4 \omega_{t}\left\{F_{1}\left(\frac{1}{\omega_{t}}\right)-F_{1}(0)\right\}+\ln \omega_{t}\right]
\end{aligned}
$$

and

Subtracting the pole term, setting $\mu^{\prime}=m_{Z}$, and multiplying by $e^{2} / \hat{s}^{2}$, we obtain Eq. (11d).

Similarly, the quark contribution to $A_{Z Z}^{(f)}(s)$ is

$$
\left[A_{Z Z}^{(f)}(s)\right]_{\text {quarks }}=-\frac{1}{16 \hat{c}^{2}} \sum_{q}\left[\left(1-4 \hat{s}^{2} C_{3 q} Q_{q}\right)^{2} \Pi^{V}\left(s, m_{q}, m_{q}\right)+\Pi^{A}\left(s, m_{q}, m_{q}\right)\right],
$$

where $C_{3 q}=+1(-1)$ for up (down) members of the doublet and the sum is over quarks. In the $O\left(\hat{\alpha}_{s}\right)$ part we neglect all masses other than $m_{t}$ and find

$$
\begin{aligned}
{\left[A_{Z Z}^{(f)}(s)\right]_{\mathrm{QCD}}=} & -\frac{\hat{\alpha}_{s}\left(m_{Z}\right)}{16 \pi \hat{c}^{2}} \sum_{q=1}^{4}\left[\left(1-4 \hat{s}^{2} C_{3 q} Q_{q}\right)^{2}+1\right] \Pi_{1}^{V}(s, 0,0) \\
& -\frac{\hat{\alpha}_{s}\left(m_{t}\right)}{16 \pi \hat{c}^{2}}\left\{\left(1-\frac{8}{3} \hat{s}^{2}\right)^{2} \Pi_{1}^{V}\left(s, m_{t}, m_{t}\right)+\Pi_{1}^{A}\left(s, m_{t}, m_{t}\right)+\left[\left(1-\frac{4}{3} \hat{s}^{2}\right)^{2}+1\right] \Pi_{1}^{V}(s, 0,0)\right\}
\end{aligned}
$$

where the first and second terms are the contributions of the light and $(t, b)$ isodoublets, respectively. Combining Eqs. (A10) and (A14) and recalling Eqs. (11d) and (A2)-(A4), we obtain

$$
\begin{aligned}
\frac{1}{m_{Z}^{2}} \operatorname{Re}\left[\frac{A_{W W}^{(f)}\left(m_{W}^{2}\right)}{\hat{c}^{2}}-A_{Z Z}^{(f)}\left(m_{Z}^{2}\right)\right]_{\mathrm{QCD}} \\
=\frac{\hat{\alpha}_{s}\left(m_{Z}\right)}{8 \pi^{3} \hat{c}^{2}}\left[c^{2} \ln c^{2}+\left(\frac{1}{n-4}+\ln \frac{m_{Z}^{2}}{\mu^{\prime 2}}+4 \zeta(3)-\frac{55}{12}\right)\left(-s^{2}+2 \hat{s}^{2}\left(1-\frac{10}{9} \hat{s}^{2}\right)\right)\right] \\
\quad+\frac{\hat{\alpha}_{s}\left(m_{t}\right)}{16 \pi^{3} \hat{c}^{2}}\left\{c^{2} \ln c^{2}+\left(\frac{1}{n-4}+\ln \frac{m_{Z}^{2}}{\mu^{\prime 2}}+4 \zeta(3)-\frac{55}{12}\right)\left(-s^{2}+2 \hat{s}^{2}\left(1-\frac{10}{9} \hat{s}^{2}\right)\right)\right. \\
\left.\quad-c^{2}\left(4 \omega_{t} F_{1}\left(\frac{1}{\omega_{t}}\right)-\ln \omega_{t}\right)+\left(1-\frac{8}{3} \hat{s}^{2}\right)^{2}\left(\mu_{t} V_{1}\left(\frac{1}{4 \mu_{t}}\right)-\frac{\ln \mu_{t}}{4}\right)+\mu_{t} A_{1}\left(\frac{1}{4 \mu_{t}}\right)-\frac{\ln \mu_{t}}{4}\right\} .
\end{aligned}
$$

Subtracting again the pole terms, setting $\mu^{\prime}=m_{Z}$, and multiplying by $e^{2} / \hat{s}^{2}$, we obtain Eq. (15d).

Finally, in order to implement the decoupling of the top quark, we need the quantity $(\alpha / \pi) d$, where $d$ is defined in Eq. (9b). From Ref. [41] we find

$$
A_{\gamma Z}^{(t)}(s)=-\frac{\hat{e}^{2}}{3 \hat{s} \hat{c}}\left(\frac{1}{2}-\frac{4}{3} \hat{s}^{2}\right) \Pi^{V}\left(s, m_{t}, m_{t}\right),
$$

and, therefore,

$\frac{\alpha}{\pi} d=-\left.\frac{e^{2}}{3}\left(\frac{1}{2 \hat{s}^{2}}-\frac{4}{3}\right)\left(\Pi^{V}\right)^{\prime}\left(0, m_{t}, m_{t}\right)\right|_{\overline{\mathrm{MS}}}$.
The $O\left(\hat{\alpha}_{s}\right)$ part of Eq. (A17) is evaluated as in the case of $\Pi_{\gamma \gamma}^{(t)}(0)$ [cf. the discussion after Eq. (A7)]. A relevant combination that occurs in $\Delta \hat{r}_{W}$ and $\Delta \hat{r}$ is

$$
\begin{aligned}
\left.e^{2} \Pi_{\gamma \gamma}^{(t)}(0)\right|_{\overline{\mathrm{MS}}}-\frac{\alpha}{\pi} d & =\left.\frac{e^{2}}{6 \hat{s}^{2}}\left(\Pi^{V}\right)^{\prime}\left(0, m_{t}, m_{t}\right)\right|_{\overline{\mathrm{MS}}} \\
& =-\frac{\alpha}{\pi} \hat{d}
\end{aligned}
$$

where $\hat{d}$ is defined in Eq. (10d). 


\section{APPENDIX B}

In this appendix we outline the derivation of the expressions for $\Delta \hat{r}_{W}$ [cf. Eq. (10a)] and $\Delta \hat{r}$ [cf. Eq. (14)], corresponding to the strategy in which the decoupling of the top quark is implemented. As $\hat{s}^{2}\left(1-\Delta \hat{r}_{W}\right)$ and $\hat{s}^{2} \hat{c}^{2}(1-\Delta \hat{r})$ are physical observables [cf. Eqs. (2) and (3)], we define $\Delta \hat{r}_{W}$ and $\Delta \hat{r}$ in such a way that

$$
\begin{aligned}
& {\left[\hat{s}^{2}\left(1-\Delta \hat{r}_{W}\right)\right]_{\text {old }}=\left[\hat{s}^{2}\left(1-\Delta \hat{r}_{W}\right)\right]_{\text {new }},} \\
& {\left[\hat{s}^{2} \hat{c}^{2}(1-\Delta \hat{r})\right]_{\text {old }}=\left[\hat{s}^{2} \hat{c}^{2}(1-\Delta \hat{r})\right]_{\text {new }} .}
\end{aligned}
$$

Here the subscript "old" labels the quantities obtained when the $\overline{\mathrm{MS}}$ counterterms cancel only the divergent parts involving $\delta$ [cf. Eq. (5a)], as in Ref. [1], while "new" denotes the corrections employed in the present paper, where the $\overline{\mathrm{MS}}$ counterterms contain small finite parts necessary to implement the top-quark decoupling in relevant amplitudes. Our strategy is to retain, in Eqs. (B1) and (B2), terms of $O\left(\alpha^{2}\right)$ when they involve large logarithmic or $m_{t}^{2} / m_{Z}^{2}$ enhancements, but to neglect them otherwise. We also neglect certain corrections of $O\left(\alpha^{3}\right)$. Recalling $e_{0}^{2}=\hat{e}^{2} / \hat{Z}_{e}$ and Eqs. (7d), (9a), (9b,) and (A18), we have

$$
\begin{aligned}
\hat{s}_{\text {old }}^{2} & =\hat{s}_{\text {new }}^{2}\left(1+\frac{\hat{\alpha}}{\pi} d\right), \\
{\left[\frac{\hat{e}^{2}}{\hat{s}^{2}}\right]_{\text {old }} } & =\left[\frac{\hat{e}^{2}}{\hat{s}^{2}}\right]_{\text {new }}\left(1-\frac{\hat{\alpha}}{\pi} \hat{d}\right),
\end{aligned}
$$

where $d$ and $\hat{d}$ are defined in Eqs. (9c) and (10d), respectively.

The derivation of Eq. (10a) follows almost immediately. Inserting Eq. (B3) into Eq. (B1), we find

$$
\left(1+\frac{\hat{\alpha}}{\pi} d\right)\left[1-\Delta \hat{r}_{W}\right]_{\text {old }}=\left[1-\Delta \hat{r}_{W}\right]_{\text {new }} \text {. }
$$

As the only large correction in $\Delta \hat{r}_{W}$ is $(\alpha / \pi) \Delta_{\gamma}$ and $\hat{\alpha}(1-$ $\left.\alpha \Delta_{\gamma} / \pi\right)=\alpha$ [cf. Eq. (8c)], we obtain

$$
\left(\Delta \hat{r}_{W}\right)_{\text {new }}=\left(\Delta \hat{r}_{W}\right)_{\text {old }}-\frac{\alpha}{\pi} d
$$

The expression for $\left(\Delta \hat{r}_{W}\right)_{\text {old }}$ in terms of $\Pi_{\gamma \gamma}^{(f)}(0), W W$ self-energies, and the vertex- and box-diagram corrections to $\mu$ decay, is given in Eqs. (7b) and (8b) of Ref. [1]. The only contribution to $\left(\Delta \hat{r}_{W}\right)$ old with large logarithmic enhancement is $\left.e^{2} \Pi_{\gamma \gamma}^{(f)}(0)\right|_{\overline{\mathrm{MS}}}$ and, to $O(\alpha)$, this does not involve $\hat{s}^{2}$. Therefore, we can replace $\hat{s}_{\text {old }}^{2} \rightarrow \hat{s}_{\text {new }}^{2}$ everywhere in $\left(\Delta \hat{r}_{W}\right)_{\text {old }}$, neglecting very small terms of $O\left(\alpha^{2}\right)$. Inserting the expression for $\left(\Delta \hat{r}_{W}\right)_{\text {old }}$ in Eq. (B6), we obtain Eq. (10a).

The derivation of Eq. (14) is more subtle. Inserting Eq. (B3) and

$$
\hat{c}_{\text {old }}^{2}=\hat{c}_{\text {new }}^{2}\left(1-\frac{\hat{\alpha}}{\pi}\left[\frac{\hat{s}^{2}}{\hat{c}^{2}}\right]_{\text {new }} d\right)
$$

into Eq. (B2), we have

$$
(\Delta \hat{r})_{\text {new }}=(\Delta \hat{r})_{\text {old }}-\frac{\hat{\alpha}}{\pi} d\left(1-\left[\frac{\hat{s}^{2}}{\hat{c}^{2}}\right]_{\text {new }}\right)[1-\Delta \hat{r}]_{\text {old }} .
$$

We first consider the second term in Eq. (B8). Using Eq. (18b), this contribution can be written as

$$
-(\hat{\alpha} / \pi) d\left(1-\left[\hat{s}^{2} / \hat{c}^{2}\right]_{\text {new }}\right)\left[1-\Delta \hat{r}_{W}\right]_{\text {old }} c^{2} / \hat{c}_{\text {old }}^{2},
$$

which, neglecting small $O\left(\alpha^{2}\right)$ terms, becomes

$$
-(\alpha / \pi) d\left(1-\left[\hat{s}^{2} / \hat{c}^{2}\right]_{\text {new }}\right) c^{2} / \hat{c}_{\text {new }}^{2}
$$

Next we analyze the first term on the rhs of Eq. (B8). According to Eq. (15b) of Ref. [1], it is given by

$$
(\Delta \hat{r})_{\text {old }}=\left(\Delta \hat{r}_{W}\right)_{\text {old }}-\left[\frac{\hat{e}^{2}}{\hat{s}^{2} \hat{c}^{2}}\left(1-\Delta \hat{r}_{W}\right)\right]_{\text {old }} \operatorname{Re}\left[\frac{A_{W W}\left(m_{W}^{2}\right)-\hat{c}^{2} A_{Z Z}\left(m_{Z}^{2}\right)}{m_{Z}^{2}}\right]_{\overline{\mathrm{MS}}}^{\text {old }}
$$

Examination of Eq. (A.8) of Ref. [1] shows that

$$
\operatorname{Re}\left[\frac{A_{W W}\left(m_{W}^{2}\right)-\hat{c}^{2} A_{Z Z}\left(m_{Z}^{2}\right)}{m_{Z}^{2}}\right]_{\overline{\mathrm{MS}}}^{\mathrm{old}}=\frac{1}{8 \pi^{2}}\left(\frac{3 m_{t}^{2}}{8 m_{Z}^{2}}+\frac{1}{2} \ln \frac{m_{t}}{m_{Z}}+\cdots\right),
$$

where the ellipses represent nonleading terms [some of which involve $\ln \left(m_{t} / m_{Z}\right)$ with very small coefficients proportional to $s^{2}, \hat{s}^{2}$, or $\hat{s}^{4}$ ]. The significant point is that the leading contributions in Eq. (B10) are independent of $\hat{s}^{2}$. Therefore, in Eq. (B9) we can replace the last factor in the second term by an analogous expression with $\hat{s}_{\text {old }}^{2} \rightarrow \hat{s}_{\text {new }}^{2}$, the difference being again small terms of $O\left(\alpha^{2}\right)$. Using Eqs. (B4), (B6), and (B7), Eq. (B9) becomes

$$
(\Delta \hat{r})_{\text {old }}=\left(\Delta \hat{r}_{W}\right)_{\text {new }}+\frac{\alpha}{\pi} d-\left[\frac{\hat{e}^{2}}{\hat{s}^{2}}\right]_{\text {new }} \frac{1}{m_{Z}^{2}}\left[1-\Delta \hat{r}_{W}-\frac{\alpha}{\pi} \hat{d}-\frac{\alpha}{\pi} d\left(1-\frac{\hat{s}^{2}}{\hat{c}^{2}}\right)\right]_{\text {new }} \operatorname{Re}\left[\frac{A_{W W}\left(m_{W}^{2}\right)}{\hat{c}^{2}}-A_{Z Z}\left(m_{Z}^{2}\right)\right]_{\overline{\mathrm{MS}}}^{\text {new }}
$$

Recalling Eqs. (10), (12b), and (15a) of Ref. [1] and neglecting again very small contributions of $O\left(\alpha^{2}, \alpha^{3}\right)$, we find that the term proportional to $(\alpha / \pi) d\left(1-\hat{s}^{2} / \hat{c}^{2}\right)$ in Eq. (B11) can be written as $(\alpha / \pi) d\left(1-\hat{s}^{2} / \hat{c}^{2}\right)\left(c^{2} / \hat{c}^{2}-1\right)$. Inserting the above results into Eq. (B8), we find 


$$
\begin{aligned}
(\Delta \hat{r})_{\text {new }}= & \left(\Delta \hat{r}_{W}\right)_{\text {new }}+\frac{\alpha}{\pi} d\left[1+\left(1-\frac{\hat{s}^{2}}{\hat{c}^{2}}\right)\left(\frac{c^{2}}{\hat{c}^{2}}-1\right)-\left(1-\frac{\hat{s}^{2}}{\hat{c}^{2}}\right) \frac{c^{2}}{\hat{c}^{2}}\right]_{\text {new }} \\
& -\left[\frac{\hat{e}^{2}}{\hat{s}^{2}}\right]_{\text {new }} \frac{1}{m_{Z}^{2}}\left(1-\left(\Delta \hat{r}_{W}\right)_{\text {new }}-\frac{\alpha}{\pi} \hat{d}\right) \operatorname{Re}\left[\frac{A_{W W}\left(m_{W}^{2}\right)}{\hat{c}^{2}}-A_{Z Z}\left(m_{Z}^{2}\right)\right]_{\overline{\mathrm{MS}}}^{\mathrm{new}}
\end{aligned}
$$

The coefficient of $(\alpha / \pi) d$ simplifies to $\hat{s}^{2} / \hat{c}^{2}$ and Eq. (B12) equals Eq. (14).

\section{APPENDIX C}

If terms proportional to squares of mixing angles are neglected, the fermionic contributions to the $W W$ self-energy become a sum over independent isodoublet contributions and we obtain

$$
\begin{aligned}
B_{0}^{(f)}=\frac{\alpha}{12 \pi \hat{s}^{2}} & \sum_{\text {doublets }} N_{c} f_{1}\left(\omega_{+}, \omega_{-}\right) \\
f_{1}\left(\omega_{+}, \omega_{-}\right)= & \ln \left[c^{2}\left(\omega_{+} \omega_{-}\right)^{1 / 2}\right]+\left[1-\frac{\omega_{+}+\omega_{-}}{2}-\frac{\left(\omega_{+}-\omega_{-}\right)^{2}}{2}\right] 2 \Omega\left(\omega_{+}, \omega_{-}\right) \\
& +\frac{3\left(\omega_{+}^{2}+\omega_{-}^{2}\right)-\left(\omega_{+}-\omega_{-}\right)^{4}}{4\left(\omega_{+}-\omega_{-}\right)} \ln \frac{\omega_{+}}{\omega_{-}}-\frac{5}{3}+\frac{\omega_{+}+\omega_{-}}{4}+\frac{\left(\omega_{+}-\omega_{-}\right)^{2}}{2}
\end{aligned}
$$

where $B_{0}^{(f)}$ is defined in Eqs. (11a) and (11b), $N_{c}=3$ and $N_{c}=1$ for quark and lepton isodoublets, respectively, and $\omega_{ \pm}=m_{ \pm}^{2} / m_{W}^{2}$, with $m_{+}$and $m_{-}$being the masses of the "up" and "down" fermions in the isodoublet. Calling

$$
C \equiv \frac{\omega_{+}+\omega_{-}}{2}-\frac{\left(\omega_{+}-\omega_{-}\right)^{2}}{4}-\frac{1}{4}
$$

the function $\Omega\left(\omega_{+}, \omega_{-}\right)$is given by

$$
\begin{array}{ll}
\Omega\left(\omega_{+}, \omega_{-}\right)=C^{1 / 2} \cos ^{-1} \frac{\omega_{+}+\omega_{-}-1}{2\left(\omega_{+} \omega_{-}\right)^{1 / 2}} & \text { for } C>0, \\
\Omega\left(\omega_{+}, \omega_{-}\right)=\frac{|C|^{1 / 2}}{2} \ln \left|\frac{\omega_{+}+\omega_{-}-1-2|C|^{1 / 2}}{\omega_{+}+\omega_{-}-1+2|C|^{1 / 2}}\right| & \text { for } C<0 .
\end{array}
$$

Equations (C4) and (C5) are a simpler version of Eqs. (A10) and (A11) of Ref. [49]. As expected from the integral representation of the self-energies $[41], f_{1}\left(\omega_{+}, \omega_{-}\right)$is a symmetric function of $\omega_{+}$and $\omega_{-}$. As $\omega_{-} \rightarrow 0$,

$$
f_{1}\left(\omega_{+}, 0\right)=\ln \left(c^{2} \omega_{+}\right)+\left(\omega_{+}-1\right)^{2}\left(1+\frac{\omega_{+}}{2}\right) \ln \left|\frac{\omega_{+}-1}{\omega_{+}}\right|-\frac{5}{3}+\frac{\omega_{+}}{4}+\frac{\omega_{+}^{2}}{2},
$$

while in the limit $\omega_{+}, \omega_{-} \rightarrow 0$,

$$
f_{1}(0,0)=\ln c^{2}-\frac{5}{3}
$$

If all the fermion masses other than $m_{t}$ are neglected, Eqs. (C1), (C6), and (C7) lead to Eq. (11c). In order to estimate the terms of $O\left(m_{b}^{2} / m_{W}^{2}\right)$, we consider the case $\omega_{+}>1$ and $\omega_{-} \ll 1$. Including corrections of $O\left(\omega_{-}\right)$, we have

$$
f_{1}\left(\omega_{+}, \omega_{-}\right)=f_{1}\left(\omega_{+}, 0\right)+\frac{3}{2} \omega_{-}\left[\left(1+\omega_{+}^{2}\right) \ln \frac{\omega_{+}}{\omega_{+}-1}-\omega_{+}-\frac{1}{2}\right]+O\left(\omega_{-}^{2}\right) .
$$

Inserting the second term of Eq. (C8) in the $(t, b)$-isodoublet contribution to Eq. (C1), and identifying $\omega_{-}=\omega_{b}=$ $m_{b}^{2} / m_{W}^{2}$ and $\omega_{+}=\omega_{t}=m_{t}^{2} / m_{W}^{2}$, we obtain a very small shift,

$$
\left[\delta B_{0}^{(f)}\right]_{m_{b}}=\frac{3 \alpha}{8 \pi \hat{s}^{2}} \omega_{b}\left[\left(1+\omega_{t}^{2}\right) \ln \frac{\omega_{t}}{\omega_{t}-1}-\omega_{t}-\frac{1}{2}\right] .
$$

Using $m_{b}=4.5 \mathrm{GeV}$ and $m_{Z}=91.187 \mathrm{GeV}$, we find that the $O\left(m_{b}^{2} / m_{W}^{2}\right)$ corrections to Eq. $(\mathrm{C} 1)$ are $\approx 2.5 \times 10^{-5}$, $5.4 \times 10^{-6}, 2.8 \times 10^{-6}, 1.7 \times 10^{-6}$ for $m_{t}=m_{Z}, 150,200,250 \mathrm{GeV}$, respectively. We note that the cofactor of $\omega_{b}$ in Eq. (C9) tends to 0 as $m_{t} \rightarrow \infty$. To estimate the contributions of $O\left(m_{c}^{2} / m_{W}^{2}\right)$, we set $m_{s}=0$ and keep terms of $O\left(\omega_{+}\right)$for $\omega_{+} \ll 1$ in Eq. (C6),

$$
f_{1}\left(\omega_{+}, 0\right)=f_{1}(0,0)+\frac{3}{2} \omega_{+}\left(\ln \omega_{+}-\frac{1}{2}\right)+O\left(\omega_{+}^{2}\right)
$$


Inserting the second term of Eq. (C10) in the $(c, s)$-isodoublet contribution to Eq. (C1), and identifying $\omega_{+}=\omega_{c}=$ $m_{c}^{2} / m_{W}^{2}$, we obtain

$$
\left[\delta B_{0}^{(f)}\right]_{m_{c}}=\frac{3 \alpha}{8 \pi \hat{s}^{2}} \omega_{c}\left(\ln \omega_{c}-\frac{1}{2}\right) .
$$

For $m_{c}=1.5 \mathrm{GeV}$, this amounts to a shift of $-1.1 \times 10^{-5}$ to Eq. (C1). For the corrections of $O\left(m_{\tau}^{2} / m_{W}^{2}\right)$, we replace $\omega_{c} \rightarrow \omega_{\tau}=m_{\tau}^{2} / m_{W}^{2}$ and divide by the color factor 3 in Eq. (C11). This leads to a further correction of $-5 \times 10^{-6}$ to Eq. (C1).

Turning our attention to $C_{0}^{(f)}$, defined in Eqs. (15a) and (15b), and neglecting again the squares of mixing angles, we have

$$
\begin{aligned}
C_{0}^{(f)}=\frac{\alpha}{12 \pi \hat{s}^{2} \hat{c}^{2}}\left[c^{2} \sum_{\text {doublets }} N_{c} f_{2}\left(\omega_{+}, \omega_{-}\right)-\frac{1}{4} \sum_{f} N_{c} g\left(\mu_{f}\right)\right] \\
f_{2}\left(\omega_{+}, \omega_{-}\right)=\left[1-\frac{3}{2}\left(\omega_{+}+\omega_{-}\right)\right] \ln \left[c^{2}\left(\omega_{+} \omega_{-}\right)^{1 / 2}\right]+\left[1-\frac{\omega_{+}+\omega_{-}}{2}-\frac{\left(\omega_{+}-\omega_{-}\right)^{2}}{2}\right] 2 \Omega\left(\omega_{+}, \omega_{-}\right) \\
-\frac{\left(\omega_{+}-\omega_{-}\right)^{3}}{4} \ln \frac{\omega_{+}}{\omega_{-}}-\frac{5}{3}+\omega_{+}+\omega_{-}+\frac{\left(\omega_{+}-\omega_{-}\right)^{2}}{2}, \\
g\left(\mu_{f}\right)=\left[\left(1-4 \hat{s}^{2} C_{3 f} Q_{f}\right)^{2}+1\right]\left[\ln \mu_{f}+\frac{1}{3}+2\left(1+2 \mu_{f}\right)\left[\Lambda\left(D_{f}\right)-1\right]\right]-6 \mu_{f}\left[\ln \mu_{f}+2 \Lambda\left(D_{f}\right)-2\right],
\end{aligned}
$$

where the $f$ sum is over quark and lepton flavors, $N_{c}$ is again the color factor, $Q_{f}$ is the electric charge in units of the positron charge $e, C_{3 f}=+1$ (-1) for up (down) members of the doublet, $\mu_{f}=m_{f}^{2} / m_{Z}^{2}, D_{f}=4 \mu_{f}-1$, and

$$
\begin{array}{ll}
\Lambda\left(D_{f}\right)=D_{f}^{1 / 2} \tan ^{-1}\left(D_{f}^{-1 / 2}\right) & \text { for } D_{f}>0 \\
\Lambda\left(D_{f}\right)=\frac{\left|D_{f}\right|^{1 / 2}}{2} \ln \left|\frac{1+\left|D_{f}\right|^{1 / 2}}{1-\left|D_{f}\right|^{1 / 2}}\right| & \text { for } D_{f}<0
\end{array}
$$

As is clear from their structure, the contributions involving $f_{2}$ and $g$ in Eq. (C12) arise from the first and second terms in Eq. (15a), respectively.

One readily finds the limiting values

$$
\begin{aligned}
f_{2}\left(\omega_{+}, 0\right) & =\left(1-\frac{3}{2} \omega_{+}\right) \ln \left(c^{2} \omega_{+}\right)+\left(\omega_{+}-1\right)^{2}\left(1+\frac{\omega_{+}}{2}\right) \ln \left|\frac{\omega_{+}-1}{\omega_{+}}\right|-\frac{5}{3}+\omega_{+}+\frac{\omega_{+}^{2}}{2}, \\
f_{2}(0,0) & =\ln c^{2}-\frac{5}{3} \\
g(0) & =-\frac{5}{3}\left[\left(1-4 \hat{s}^{2} C_{3 f} Q_{f}\right)^{2}+1\right] .
\end{aligned}
$$

If the fermion masses other that $m_{t}$ are neglected, Eqs. (C12), (C14), and (C17)-(C19) lead to Eq. (15c). To discuss the corrections of $O\left(m_{b}^{2} / m_{W}^{2}\right)$, we consider $f_{2}\left(\omega_{+}, \omega_{-}\right)$for $\omega_{+}>1, \omega_{-} \ll 1$ and $g\left(\mu_{f}\right)$ for $\mu_{f} \ll 1$. One readily finds

$$
\begin{aligned}
f_{2}\left(\omega_{+}, \omega_{-}\right) & =f_{2}\left(\omega_{+}, 0\right)+\frac{3}{2} \omega_{-}\left[\left(1+\omega_{+}^{2}\right) \ln \frac{\omega_{+}}{\omega_{+}-1}-\omega_{+}-\ln \left(c^{2} \omega_{+}\right)\right]+O\left(\omega_{-}^{2}\right), \\
g\left(\mu_{f}\right) & =g(0)-48 \mu_{f} \hat{s}^{2} Q_{f}\left(2 \hat{s}^{2} Q_{f}-C_{3 f}\right)+O\left(\mu_{f}^{2}\right) .
\end{aligned}
$$

Identifying $\omega_{-}=\omega_{b}, \omega_{+}=\omega_{t}, \mu_{f}=\mu_{b}=m_{b}^{2} / m_{Z}^{2}$ and inserting Eqs. (C20) and (C21) into Eq. (C12), we find for the leading correction of $O\left(\mu_{b}\right)$

$$
\left[\delta C_{0}^{(f)}\right]_{m_{b}}=\frac{3 \alpha}{8 \pi \hat{s}^{2} \hat{c}^{2}} \mu_{b}\left[\left(1+\omega_{t}^{2}\right) \ln \frac{\omega_{t}}{\omega_{t}-1}-\omega_{t}-\ln \left(c^{2} \omega_{t}\right)-\frac{8}{3} \hat{s}^{2}\left(1-\frac{2}{3} \hat{s}^{2}\right)\right] .
$$

Using the same input values as in Eq. (C9), we find that the $O\left(m_{b}^{2} / m_{W}^{2}\right)$ corrections to Eq. (C12) are $\approx 2.5 \times 10^{-5}$, $-6.7 \times 10^{-6},-1.6 \times 10^{-5},-2.3 \times 10^{-5}$ for $m_{t}=m_{Z}, 150,200,250 \mathrm{GeV}$, respectively. To estimate the corrections of $O\left(m_{c}^{2} / m_{W}^{2}\right)$, we set $\omega_{-}=0$ and keep terms of $O\left(\omega_{+}\right)$for $\omega_{+} \ll 1$ in Eq. (C17). In conjunction with Eq. (C21), this gives 


$$
\left[\delta C_{0}^{(f)}\right]_{m_{c}}=\frac{3 \alpha}{8 \pi \hat{s}^{2} \hat{c}^{2}} \mu_{c}\left[\frac{16}{3} \hat{s}^{2}\left(\frac{4}{3} \hat{s}^{2}-1\right)-\ln c^{2}\right]
$$

where $\mu_{c}=m_{c}^{2} / m_{Z}^{2}$. For $m_{c}=1.5 \mathrm{GeV}$, this is $\approx-8 \times 10^{-7}$. The corrections of $O\left(m_{\tau}^{2} / m_{W}^{2}\right)$ are even smaller on account of the absence of the color factor.

There are also very small corrections of $O\left(m_{b}^{2} / m_{Z}^{2}\right)$ and $O\left(m_{c}^{2} / m_{Z}^{2}\right)$ associated with the $f_{1}\left(r_{q}\right)$ contribution to $e^{2} \Pi_{\gamma \gamma}^{(f)}(0)$ [cf. Eq. (7a)]. Their magnitude is $\approx+5 \times 10^{-6}$.

Putting all these small $O\left(m_{f}^{2} / m_{W}^{2}\right)$ corrections together, we see that for $m_{t}=m_{Z}, 150,200,250 \mathrm{GeV}$ they amount to $(1.4,-0.6,-0.8,-0.9) \times 10^{-5}$ in the case of $\Delta \hat{r}_{W}$ and $(-1.0,+0.2,+0.9,+1.5) \times 10^{-5}$ in the case of $\Delta \hat{r}$. On the other hand, $\Delta r=\Delta \hat{r}_{W}-\left(\hat{c}^{2} / s^{2}\right)\left(\Delta \hat{r}_{W}-\Delta \hat{r}\right)$ and the small corrections are enhanced in the second term, leading to shifts of $(-6.5,+2.1,+5.1,+7.8) \times 10^{-5}$ for the same values of $m_{t}$.

[1] G. Degrassi, S. Fanchiotti, and A. Sirlin, Nucl. Phys. B351, 49 (1991).

[2] A. Sirlin, Phys. Rev. D 22, 971 (1980).

[3] A. Sirlin, Phys. Rev. D 29, 89 (1984).

[4] M. Consoli, W. Hollik, and F. Jegerlehner, Phys. Lett. B 227, 167 (1989).

[5] G. Burgers, F. Jegerlehner, B. Kniehl, and J. Kühn, in $Z$ Physics at LEP 1, Proceedings of the Workshop, Geneva, Switzerland, 1989, edited by G. Altarelli, R. Kleiss, and C. Verzegnassi (CERN Report No. 89-08, Geneva, 1989), Vol. 1, p. 55.

[6] J. J. van der Bij and F. Hoogeveen, Nucl. Phys. B283, 477 (1987).

[7] R. Barbieri, M. Beccaria, P. Ciafaloni, G. Curci, and A. Viceré, Phys. Lett. B 288, 95 (1992); M. Beccaria (private communication).

[8] S. Fanchiotti and A. Sirlin, in Bég Memorial Volume, edited by A. Ali and P. Hoodbhoy (World Scientific, Singapore, 1991), p. 58.

[9] H. Burkhardt, F. Jegerlehner, G. Penso, and C. Verzegnassi, Z. Phys. C 43, 497 (1989).

[10] F. Jegerlehner, in Proceedings of the 1990 Theoretical Advanced Study Institute in Elementary Particle Physics, edited by P. Langacker and M. Cvetič (World Scientific, Singapore, 1991), p. 476.

[11] A. Sirlin, Phys. Lett. B 232, 123 (1989).

[12] S. Fanchiotti and A. Sirlin, Phys. Rev. D 41, 319 (1990).

[13] A. Sirlin, Phys. Rev. Lett. 67, 2127 (1991); Phys. Lett. B 267, 240 (1991).

[14] M. Consoli and A. Sirlin, in Physics at LEP, LEP Jamboree, Geneva, Switzerland, 1985, edited by J. Ellis and R. Peccei (CERN Report No. 86-02, Geneva, 1986), Vol. 1, p. 63.

[15] S. Willenbrock and G. Valencia, Phys. Lett. B 259, 373 (1991); R. G. Suart, ibid. 262, 113 (1991); 272, 353 (1991); T. Bhattacharya and S. Willenbrock, Phys. Rev. D 47, 4022 (1993).

[16] R. Tanaka, in Proceedings of the XXVI International Conference on High Energy Physics, Dallas, Texas, 1992, edited by J. Sanborn, AIP Conf. Proc. No. 272 (AIP, New York, 1993).

[17] G. Degrassi and A. Sirlin, Nucl. Phys. B352, 342 (1991).

[18] U. Amaldi et al., Phys. Rev. D 36, 1385 (1987); G. Costa, J. Ellis, G. L. Fogli, D. V. Nanopoulos, and F. Zwirner, Nucl. Phys. B297, 244 (1988).

[19] P. Langacker, M. Luo, and A. Mann, Rev. Mod. Phys. 64, 87 (1992).

[20] G. Altarelli, R. Barbieri, and S. Jadach, Nucl. Phys.
B369, 3 (1992).

[21] P. Langacker, in Electroweak Interactions and Unified Theories, Proceedings of the 27th Rencontres de Moriond, Les Arcs, France, 1992 (unpublished).

[22] A. Djouadi and C. Verzegnassi, Phys. Lett. B 195, 265 (1987); A. Djouadi, Nuovo Cimento A 100, 357 (1988).

[23] T. H. Chang, K. J. F. Gaemers, and W. L. van Neerven, Nucl. Phys. B202, 407 (1982).

[24] B. A. Kniehl, J. H. Kühn, and R. G. Stuart, Phys. Lett. B 214, 621 (1988); also in Polarization at LEP, edited by G. Alexander et al. (CERN Report No. 88-06, Geneva, Switzerland, 1988), Vol. 1, p. 158; B. A. Kniehl, Comput. Phys. Commun. 58, 293 (1990).

[25] B. A. Kniehl, Nucl. Phys. B347, 86 (1990).

[26] F. Halzen and B. A. Kniehl, Nucl. Phys. B353, 567 (1991).

[27] V. S. Fadin and V. A. Khoze, Pis'ma Zh. Eksp. Teor. Fiz. 46, 417 (1987) [JETP Lett. 46, 525 (1987)]; Yad. Fiz. 48, 487 (1988) [Sov. J. Nucl. Phys. 48, 309 (1988)].

[28] M. J. Strassler and M. E. Peskin, Phys. Rev. D 43, 1500 (1991).

[29] W. Kwong, Phys. Rev. D 43, 1488 (1991).

[30] B. A. Kniehl and A. Sirlin, Nucl. Phys. B371, 141 (1992).

[31] B. A. Kniehl and A. Sirlin, Phys. Rev. D 47, 883 (1993).

[32] W. J. Marciano and J. L. Rosner, Phys. Rev. Lett. 65, 2963 (1990).

[33] W. J. Marciano, Annu. Rev. Nucl. Part. Sci. 41, 469 (1991).

[34] W. A. Bardeen, A. J. Buras, D. W. Duke, and T. Muta, Phys. Rev. D 18, 3998 (1978); A. J. Buras, Rev. Mod. Phys. 52, 199 (1980).

[35] W. J. Marciano (private communication).

[36] CDF Collaboration, F. Abe et al., Phys. Rev. Lett. 68, 447 (1992); Phys. Rev. D 45, 3921 (1992).

[37] CDF Collaboration, F. Abe et al., Phys. Rev. Lett. 65, 2243 (1990); Phys. Rev. D 43, 2070 (1991); UA2 Collaboration, J. Alitti et al., Phys. Lett. B 276, 354 (1992).

[38] Particle Data Group, K. Hikasa et al., Phys. Rev. D 45, S1 (1992), Part II.

[39] S. Bethke, J. Phys. G 17, 1455 (1991).

[40] S. Sarantakos, A. Sirlin, and W. J. Marciano, Nucl. Phys. B217, 84 (1983).

[41] W. J. Marciano and A. Sirlin, Phys. Rev. D 22, 2695 (1980).

[42] V. A. Novikov, L. B. Okun, M. A. Shifman, A. I. Vainshtein, M. B. Voloshin, and V. I. Zakharov, Phys. Rep. C 41, 1 (1978); M. A. Shifman, A. I. Vainshtein, and V. I. Zakharov, Nucl. Phys. B147, 385 (1979). 
[43] B. Grinstein and M.-Y. Wang, Nucl. Phys. B377, 480 (1992).

[44] J. Gasser and H. Leutwyler, Phys. Rep. 87, 77 (1982).

[45] F. Jegerlehner, in Progress in Particle and Nuclear Physics, edited by A. Faessler (Pergamon, Oxford, 1991), Vol. 27, p. 1; A. Sirlin (unpublished).

[46] H. Georgi and H. D. Politzer, Phys. Rev. D 14, 1829 (1976).

[47] M. Veltman, Nucl. Phys. B123, 89 (1977); M. S. Chanowitz, M. A. Furman, and I. Hinchliffe, Phys. Lett. 78B, 285 (1978).

[48] M. Consoli, S. Lo Presti, and L. Maiani, Nucl. Phys.
B223, 474 (1983); F. Halzen, Z. Hioki, and M. Konuma, Phys. Lett. 126B, 129 (1983); Z. Hioki, Nucl. Phys. B229, 284 (1983); Phys. Rev. D 45, 1814 (1992); Mod. Phys. Lett. A 7, 1009 (1992); Tokushima Report No. 9202 (unpublished).

[49] S. Bertolini and A. Sirlin, Nucl. Phys. B248, 589 (1984).

[50] A. Sirlin, presented at the Workshop on Precise Electroweak Measurements, Santa Barbara, California, 1991 (unpublished).

[51] L. Rolandi, in Proceedings of the XXVI International Conference on High Energy Physics [16]. 